\title{
NEUTRONICS MODELING AND SIMULATION OF SHARP FOR FAST REACTOR ANALYSIS
}

\author{
W. S. YANG*, M. A. SMITH, C. H. LEE, A. WOLLABER, D. KAUSHIK and A. S. MOHAMED \\ Argonne National Laboratory \\ 9700 South Cass Avenue \\ Argonne, Illinois 60439 \\ *Corresponding author. E-mail : wyang@anl.gov
}

Received August 02, 2010

This paper presents the neutronics modeling capabilities of the fast reactor simulation system SHARP, which ANL is developing as part of the U.S. DOE's NEAMS program. We discuss the three transport solvers (PN2ND, SN2ND, and MOCFE) implemented in the UNIC code along with the multigroup cross section generation code $\mathrm{MC}^{2}-3$. We describe the solution methods and modeling capabilities, and discuss the improvement needs for each solver, focusing on massively parallel computation. We present the performance test results against various benchmark problems and ZPR-6 and ZPPR critical experiments. We also discuss weak and strong scalability results for the SN2ND solver on the ZPR-6 critical assembly benchmarks.

KEYWORDS : Neutron Transport, Simulation, Multigroup Cross Section, Fast Reactor

\section{INTRODUCTION}

Reactor design is an iterative process among various disciplines such as reactor physics, thermal fluid dynamics, mechanics, reactor and plant dynamics, fuel behavior, and instrumentation and control, and requires a wide range of modeling and simulation tools that can accurately predict key system performance and safety characteristics. Most code systems for fast reactor design were initiated more than twenty years ago and designed to accommodate the computing resources, tools and methods available at that time, although considerable relevant advances of methods and codes have been made more recently. Improvements to the available tools are thus needed to design future reactor systems that must meet performance, safety and economic goals higher than the current ones. The uncertainties and biases in the various areas of reactor design activities need to be reduced by enhanced prediction capabilities. These improvements are important to minimize the costly and lengthy procedures of building multiple representative mockup experiments to confirm the predictions.

The challenge in neutronics analysis is to efficiently generate solutions to the Boltzmann equation by taking into account the geometric complexity and complicated energy dependence of nuclear data. The Monte Carlo method can represent these details, but needs sufficiently low statistical uncertainty, reliable variance estimates and uncertainty propagation. Computing resource requirements still remain unmanageable for many types of routine design analyses, including accurate estimations of detailed pinby-pin power distributions, effects of small perturbations, thermal feedback, error propagation via fuel depletion, and transient analysis. Thus, its use is typically limited to steady-state reference solutions without thermal feedback. As a result, the current design tools rely heavily upon deterministic methods based on various approximations and sophisticated multistep procedures [1-3]. Detailed energy variable treatments are done only at the pin cell level (or using a homogeneous mixture of fuel, coolant, and structure materials) with approximate boundary conditions. Then, by performing a series of subdomain calculations with a larger problem domain but fewer modeling details, space and energy condensed parameters are defined and tabulated for global core calculations. Global analyses are performed for three-dimensional models composed of homogenized regions with low-order approximations of the Boltzmann equation (e.g., diffusion approximation). Detailed pin-by-pin information is then recovered by reconstruction (de-homogenization) methods.

In order to explore a broad range of design space and incorporate innovative design features, it is necessary to minimize approximations and increase the modeling capability from first principles. Significantly improved capabilities to simulate multiphysics phenomena are also 
required to facilitate global design optimizations. For example, in fast reactors where the coolant temperature and its rise across the core are much larger than in light water reactors, the reactivity feedback due to radial expansion of the core is one of the most important reactivity feedback phenomena [4,5]. In order to reliably evaluate this reactivity feedback effect, the geometrical deformation of assembly ducts and core structures needs to be determined accurately by consistently coupled neutronics, thermal fluids and thermo-structural analyses. With the current neutronics analysis tools based on homogenized assembly models and structured meshes, however, structural deformations across a mesh interface cannot be modeled accurately. The reactivity effect is thus not properly accounted for.

As part of the U.S. Department of Energy's Global Nuclear Energy Partnership (GNEP) program, an effort was launched in April 2007 to develop the SHARP (Simulation for High-efficiency Advanced Reactor Prototyping) [6] fast reactor simulation system. The initial plan was to develop and implement revolutionary, highfidelity reactor simulation capabilities for sodium-cooled fast reactor (SFR) design and safety analysis, with an emphasis on highly scalable simulation capabilities that can harness the power of current generation high performance computing (HPC) capacity in preparation for future high performance desktop machines. The initial plan focused on completing full-core transient simulations using the largest computing resources available (beyond exascale) at the end of the first decade of development. In October 2008, this effort was rearranged into the Reactor IPSC (Integrated Performance and Safety Code) of the Nuclear Energy Advanced Modeling and Simulation (NEAMS) program of the U.S. DOE, with emphasis on expanding its applicability from the initially targeted SFR to other envisioned advanced systems.

Under this Reactor IPSC program, the high-fidelity deterministic neutron transport code UNIC is being developed [7-9]. A long-term goal is to replace the multistep averaging approximations by progressively applying more accurate treatments of the entire space-angle-energy phase space with sufficiently fine-grained levels of discretization. Given that high-fidelity transport calculations are not required in all areas of reactor analysis, it is also desirable to develop an analysis tool that can allow the user to start at the current level of reactor analysis and transition smoothly (i.e., with familiar input/output) to less crude homogenization approaches and eventually to fully heterogeneous descriptions. In this way, the experienced reactor analyst can choose the desired level of approximation appropriate for their computational resources and analysis goals. In order to faithfully represent the reactivity feedback caused by structural deformation, we use a conforming unstructured, finite-element mesh representation of the domain. The current mesh generation tool is CUBIT [10] while all visualization of the solution is done with VisIt [11]. At present, three transport equation solvers have been developed: PN2ND, SN2ND, and MOCFE. PN2ND is based upon the second-order evenparity transport equation and implements spherical harmonics for the angular approximation. SN2ND is also based upon the second-order even-parity transport equation but a discrete ordinates approximation is applied for the angular approximation. These solvers are designed to use 100,000 processors or more for reasonably large problems with reduced homogenization approximations (e.g., pincell level). MOCFE is a method of characteristics solver for the first-order transport equation, and its targeted application is for explicit geometry, fine-group problems with the short-term purpose of cross section generation and the long-term purpose of fine-level transport calculations without homogenization. UNIC has been applied to detailed simulations of full-core mockup critical experiments on up to 295,000 processor cores. In addition to the finiteelement-based solvers above, the capability of the nodal transport code VARIANT $[12,13]$ is being rebuilt in UNIC to be applied to assembly-homogenized problems, called NODAL. NODAL is currently limited to diffusion theory and can potentially be used as one step in a multigrid preconditioner for the other solvers in UNIC.

To generate multigroup cross sections in fast reactor applications, the $\mathrm{MC}^{2}-3$ code [14] has been developed by improving the resonance self-shielding and spectrum calculation methods of $\mathrm{MC}^{2}-2$ [15] and integrating the one-dimensional cell calculation capabilities of SDX [16]. For ultrafine group spectrum calculations, to eliminate the limitations of the generalized resonance integral method of $\mathrm{MC}^{2}-2$, the resolved resonance cross sections are self shielded by numerical integrations of pointwise cross sections. The continuous slowing-down method used in the resolved resonance energy range has been replaced with the multigroup method in order to minimize the approximations involved in the slowing-down by light elements (especially hydrogen). The isotropic inelastic scattering approximation has also been removed. For the optional hyperfine group (HFG) spectrum calculation in the resolved resonance range, the isotropic source approximation has been eliminated by incorporating anisotropic scattering sources. In addition, the onedimensional transport calculation of SDX, which was previously limited to a fine group level, has been extended to ultrafine and hyperfine group levels. The $\mathrm{MC}^{2}-3$ code is being incorporated into UNIC to alleviate the approximations used in cell calculations by the use of transport solutions for extended problem domains and to generate the cross sections consistently with the material and temperature distributions used in transport calculations.

In this paper, we report on these early efforts to develop an improved neutronics modeling capability for fast reactor analysis. The solution methods and modeling capabilities of UNIC and $\mathrm{MC}^{2}-3$ are discussed in Section 2. Initial test results for various benchmark problems and critical 
experiments are presented in Section 3. The conclusions and future work are summarized in Section 4.

\section{SOLUTION METHODS AND MOELING CAPABILITIES}

The simulation of generally time-dependent, multiphysics phenomena requires solving the timedependent Boltzmann equation along with the delayed neutron precursor equations. Representing the first-order time derivative by a finite difference approximation, however, the time-dependent Boltzmann equation can be cast into the time-independent transport equation with modified absorption and source terms. Thus, for simplicity, we will discuss our methods using the steady-state neutron transport equation, which can be written in standard notation as:

$$
\begin{aligned}
& \Omega \cdot \nabla \psi(r, E, \Omega)+\Sigma_{t}(r, E) \psi(r, E, \Omega) \\
& =S_{s}(r, E, \Omega)+\frac{1}{4 \pi} S_{f}(r, E)+S_{e x}(r, E, \Omega)
\end{aligned}
$$

where $S_{s}, S_{f}$, and $S_{e x}$ are the scattering, fission and external neutron sources, respectively. The scattering and fission sources are given by

$$
\begin{gathered}
S_{s}(r, E, \Omega)=\sum_{i} N_{i}(r) \int_{E^{\prime}} d E^{\prime} \int_{4 \pi} d \Omega^{\prime} \sigma_{s}^{i} \\
\left(E^{\prime} \rightarrow E, \Omega \cdot \Omega^{\prime}\right) \psi\left(r, E^{\prime}, \Omega^{\prime}\right) \\
S_{f}(r, E)=\sum_{i} N_{i}(r) \int_{0}^{\infty} d E^{\prime} \chi^{i}\left(E^{\prime} \rightarrow E\right) v^{i} \\
\left(E^{\prime}\right) \sigma_{f}^{i}\left(E^{\prime}\right) \int_{4 \pi} d \Omega^{\prime} \psi\left(r, E^{\prime}, \Omega^{\prime}\right)
\end{gathered}
$$

where the index $i$ is used to denote the isotope dependency of scattering and fission properties. The angular distribution of scattered neutrons is typically represented as a Legendre polynomial series and thus the scattering source can be written as:

$$
\begin{aligned}
& S_{s}(r, E, \Omega)=\sum_{i} N_{i}(r) \sum_{l=0}^{L} \sum_{k=-l}^{l} \frac{2 l+1}{4 \pi} Y_{l k}(\Omega) \\
& \int_{E^{\prime}} d E^{\prime} \sigma_{s l}^{i}\left(E^{\prime} \rightarrow E\right) \int_{4 \pi} d \Omega^{\prime} Y_{l k}^{*}\left(\Omega^{\prime}\right) \psi\left(r, E^{\prime}, \Omega^{\prime}\right)
\end{aligned}
$$

Here $\sigma_{s l}^{i}$ is the $l$-th Legendre moment of the scattering kernel of isotope $i, Y_{l k}$ is the spherical harmonics, and $Y_{l k}^{*}$ is the complex conjugate of $Y_{l k}$. When there is no external source, Eq. (1) is generally cast into the form of an eigenvalue problem by dividing the fission source by the multiplication factor.

The transport equation solvers of UNIC solve the multigroup form of Eq. (1), which can be written as

$$
\begin{gathered}
\Omega \cdot \nabla \psi_{g}(r, \Omega)+\Sigma_{t g}(r) \psi_{g}(r, \Omega)=Q_{g}(r, \Omega), \\
g=1, \cdots, G
\end{gathered}
$$

For the criticality problem, the group source $Q_{g}$ can be written in terms of macroscopic cross sections as

$$
\begin{aligned}
Q_{g}(r, \Omega)= & \sum_{g^{\prime}=1}^{G} \sum_{l=0}^{L} \sum_{k=-l}^{l} \frac{2 l+1}{4 \pi} Y_{l k}(\Omega) \sum_{s l}^{g^{\prime} \rightarrow g}(r) \\
& \int_{4 \pi} d \Omega^{\prime} Y_{l k}^{*}\left(\Omega^{\prime}\right) \psi_{g^{\prime}}\left(r, \Omega^{\prime}\right) \\
& +\frac{1}{4 \pi k} \sum_{g^{\prime}=1}^{G} \chi_{g^{\prime} \rightarrow g} v \sum_{f g^{\prime}}(r) \int_{4 \pi} d \Omega^{\prime} \psi_{g^{\prime}}\left(r, \Omega^{\prime}\right)
\end{aligned}
$$

where $k$ is the system eigenvalue known as the multiplication factor. To facilitate the discussion below, we separate out the within-group scattering source and rewrite Eq. (5) as

$$
\begin{gathered}
\Omega \cdot \nabla \psi_{g}(r, \Omega)+\Sigma_{t g}(r) \psi_{g}(r, \Omega)=W_{g}(r, \Omega)+S_{g}(r, \Omega), \\
g=1, \cdots, G
\end{gathered}
$$

Here $W_{g}$ is the within-group scattering source, and $S_{g}$ includes the out-of-group scattering source and the fission neutron sources. While we are developing a Krylov subspace solver over the entire space-angle-energy system for the SN2ND and MOCFE, the bulk of the work thus far has focused on the preconditioner which requires the approximate solution of Eq. (7).

This section presents an overview of the three transport solvers implemented in UNIC for the system of equations in Eq. (7) and the methods of $\mathrm{MC}^{2}-3$ to determine the multigroup cross sections included in Eq. (7).

\subsection{PN2ND and SN2ND Solvers}

As mentioned in the introduction, both the PN2ND and SN2ND solvers are based upon the second-order even-parity transport equation. These second-order methodologies that implement continuous, spatial finite-element approximations permit the parallelization successes 
achieved in other fields with the Poisson equation (e.g., parallel conjugate gradient methods) to be used directly in UNIC. To derive the second-order methods, the angular flux in Eq. (7) is first split into even- and odd-parity components with respect to the angular variable as

$$
\psi_{g}(r, \Omega)=\psi_{g}^{+}(r, \Omega)+\psi_{g}^{-}(r, \Omega)
$$

where the superscript + denotes even parity and the superscript - denotes odd parity. Evaluating Eq. (7) for $\Omega$ and $-\Omega$, and adding and subtracting the resulting equations [17], the coupled, first-order even-parity and odd-parity equations can be obtained using Eqs. (7) and (8) as:

$$
\Omega \cdot \nabla \psi_{g}^{\mp}(r, \Omega)+\Sigma_{t g}(r) \psi_{g}^{ \pm}(r, \Omega)=W_{g}^{ \pm}(r, \Omega)+S_{g}^{ \pm}(r, \Omega)
$$

The odd-parity equation can be solved for the odd-parity flux as:

$$
\begin{aligned}
\psi_{g}^{-}(r, \Omega) & =\frac{1}{\Sigma_{t g}(r)}\left[-\Omega \cdot \nabla \psi_{g}^{+}(r, \Omega)\right. \\
& \left.+W_{g}^{-}(r, \Omega)+S_{g}^{-}(r, \Omega)\right]
\end{aligned}
$$

Substituting Eq. (10) into the even-parity equation, the second-order even-parity transport equation can be obtained as:

$$
\begin{aligned}
& -\Omega \cdot \nabla \frac{1}{\sum_{t g}(r)} \Omega \cdot \nabla \psi_{g}^{+}(r, \Omega)+\Sigma_{t g}(r) \psi_{g}^{+}(r, \Omega) \\
& =W_{g}^{+}+S_{g}^{+}-\Omega \cdot \nabla \frac{1}{\Sigma_{t g}(r)}\left(W_{g}^{-}+S_{g}^{-}\right)
\end{aligned}
$$

This differential equation is accompanied by the boundary conditions that constrain the angular flux along the boundary of the problem domain and at element interfaces.

In the PN2ND solver, the even- and odd-parity angular fluxes in Eq. (11) are respectively expanded in terms of even- and odd-parity spherical harmonic trial functions as:

$$
\psi_{g}^{+}(r, \Omega)=\sum_{l=0,2, \ldots, N-1} \sum_{k=-l}^{l} Y_{l k}(\Omega) \psi_{g l k}^{+}(r)
$$

$$
\psi_{g}^{-}(r, \Omega)=\sum_{l=1,3, \ldots, N} \sum_{k=-l}^{l} Y_{l k}(\Omega) \psi_{g l k}^{-}(r)
$$

where $N$ is the angular expansion order. Figure 1 graphically displays some selected angular trial functions. The SN2ND solver uses a discrete ordinates cubature to represent the angular variable. Because a spherical harmonics-based scattering kernel is used, the cubature on the unit sphere that results from a discrete set of directions $\Omega_{n}$ is required to satisfy the following two relationships:

$$
\sum_{n} Y_{l k}\left(\Omega_{n}\right) w_{n}=\delta_{l 0} \delta_{k 0}
$$

$$
\psi_{g l k}^{+}(r)=\sum_{n} Y_{l k}\left(\Omega_{n}\right) w_{n} \psi_{g}^{+}\left(r, \Omega_{n}\right)
$$

where $Y_{l k}$ is an even-parity spherical harmonic function and $w_{n}$ 's are the weights associated with the cubature. The $\psi_{g l k}^{+}(r)$ moments are identical to those obtained using the spherical harmonic method. Currently, Carlson's level

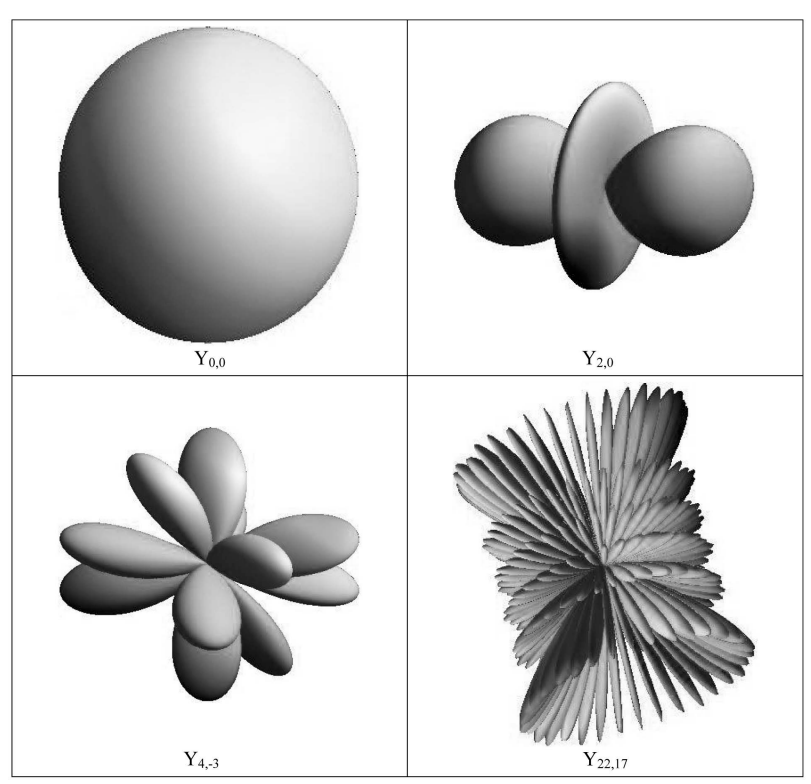

Fig. 1. Graphical Depictions of Spherical Harmonic Functions used in PN2ND 
symmetric cubature [18], the Legendre-Tchebychev product cubature [19], the Thurgood cubature [20], the LebedevLaikov cubature [21], the COBE sky cube-based cubature [22], and the Tegmark icosahedron-based cubature [23] have all been implemented into UNIC. Sample cubatures are shown in Fig. 2, where the black dots indicate the cubature abscissa intersections with the unit sphere and the coloring indicates the distribution of weights associated with the cubature.

The angular approaches are combined with a spatial finite element method [24, 25]. The problem domain is subdivided into a series of unstructured geometric elements that do not overlap, and the border of each element is defined using a series of spatial vertices which incorporate a distinct numbering scheme to facilitate computer implementations. For each type of finite element, a set of polynomial basis functions is defined within each element to satisfy the patch test. Lagrangian, serendipity, and Gauss-Lobatto polynomial trial functions are available in UNIC on six primary geometries: bar, triangle, quadrilateral, tetrahedron, triangular prism, and hexahedron. The Lagrangian, serendipity, and Gauss-Lobatto elements are available up to the ninth, second, and sixth orders, respectively. UNIC typically assumes an isoparametric geometry input, but it can easily be used on subparametric and superparametric geometric input definitions. The vertex layout for the quadratic serendipity and cubic Lagrange elements in the standard coordinate system are

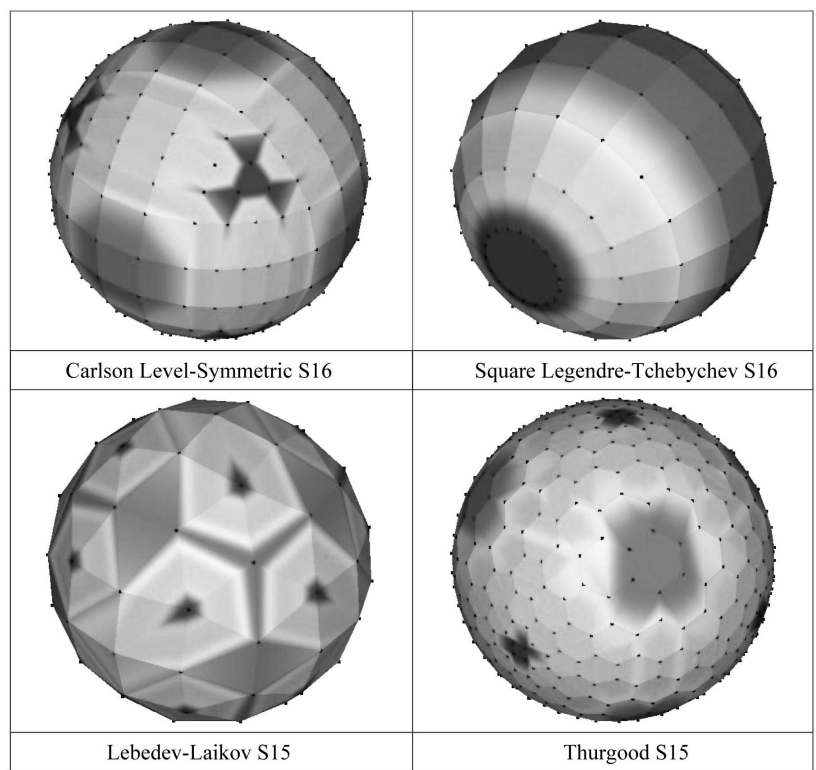

Fig. 2. Examples of Discrete Ordinates Cubatures Available in SN2ND shown in Fig. 3.

Both PN2ND and SN2ND assume a continuous finite element formulation for the even-parity transport equation and a discontinuous finite element formulation of the oddparity transport equation. This reduces Eq. (11) to a linear algebra equation for the even-parity flux $\psi_{g}^{+}$of the form:

$$
A_{g} \psi_{g}^{+}=B_{g} \psi_{g}^{+}+S_{g}^{+}+C_{g}\left(W_{g}^{-}+S_{g}^{-}\right)
$$

where $B_{g}$ represents the within-group scattering matrix. Arbitrarily oriented, reflected and vacuum boundary conditions are included along with the ability to include volumetric fixed sources. The odd-parity sources of Eq. (16) are iteratively determined with the use of Eq. (10).

The coefficient matrix $A_{g}$ is positive definite, and thus Eq. (16) can be solved efficiently using the conjugate gradient

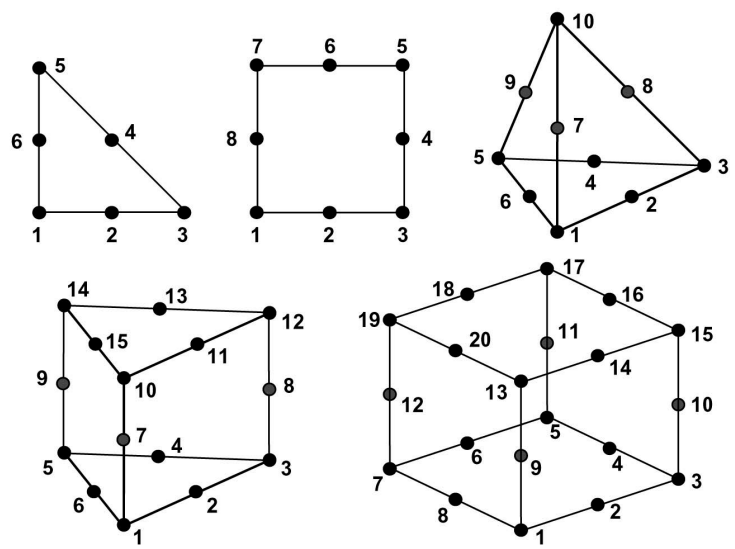

Quadratic Serendipity Elements
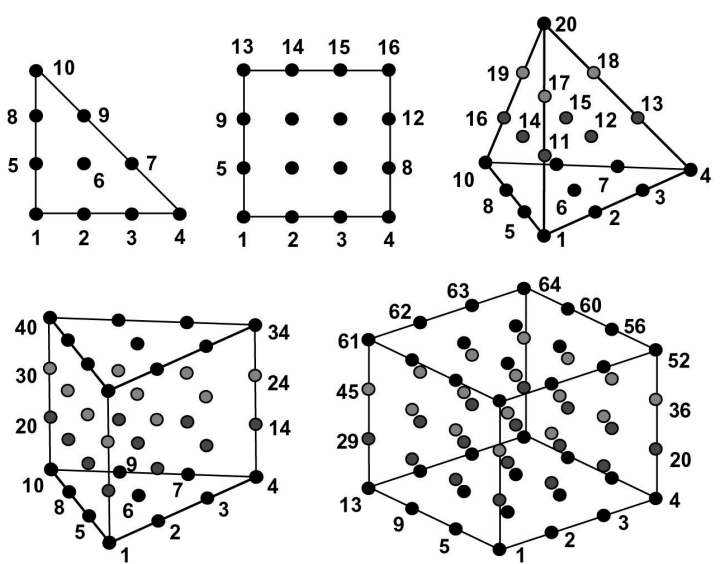

Cubic Lagrange Elements

Fig. 3. Examples of Serendipity and Lagrange Finite Elements 
method [26], when the source terms on the right hand side lagged in the iterative approach discussed below.

For PN2ND, the coefficient matrix $A_{g}$ and the withingroup scattering matrix $B_{g}$ exhibit the coupling of $\psi_{g l k}^{+}$with $\psi_{g l^{\prime} k^{\prime}}^{+}$for $l^{\prime}=l$ or $l-2$, and $k^{\prime}=k-1, k$, or $k+1$. Because these matrices have the same coupling, the within-group scattering source $W_{g}^{+}$is moved to the left hand side and $B_{g}$ is merged with $A_{g}$ in PN2ND. Reflected boundary conditions do not fundamentally change the coupling, but vacuum boundary conditions couple all angular terms together for those spatial vertices that lie along the boundary in the PN2ND coefficient matrix.

In SN2ND, no coupling occurs between the angles when no reflected boundary condition is present, and thus the $A_{g}$ matrix is block-diagonal with respect to angle. When reflected boundary conditions are present, those vertices that lie along a reflected boundary have coupling in angle, which appears as a very sparse connection in $A_{g}$. When reflected boundary conditions are present, experience has shown that the angular cubature should display the underlying symmetry in the problem, but SN2ND does not impose this behavior and can use cubatures which do not have the necessary symmetry. In general, $A_{g}$ matrix always remains nearly block diagonal with respect to angle because the number of vertices that lie upon the reflected surfaces of the domain is far fewer than the number that are internal to the domain and the implemented angular cubature generally matches the underlying symmetry of the domain. Unlike the $A_{g}$ matrix, the within-group scattering matrix $B_{g}$ is full with respect to angle. Thus Eq. (16) is solved iteratively by estimating the within-group scattering source using the most recent flux solution. While convergence of this equation is generally guaranteed, the speed of convergence strongly depends upon the ratio of scattering to collision and the amount of leakage. As such, acceleration schemes have been developed based upon the diffusion equation. For the SN2ND solver, the diffusion operator is a direct projection of the even-parity transport operator and thus the diffusion synthetic acceleration (DSA) scheme [27] is automatically fully consistent and not prone to the weakened convergence properties observed in the first-order discrete ordinates solvers.

From a parallelization point of view, $A_{g}-B_{g}$ in PN2ND and $A_{g}$ in SN2ND are both scalable operations because they can both be solved via conjugate gradient (CG) methods which have been shown to perform well on small- and largescale parallel machines. Others have reported in the literature their application of CG methods to similar equations with processor counts as high as 10,000 and with 70 to $80 \%$ efficiency. To achieve such good performance, the literature indicates that developers should create a preconditioner that contains domain decomposition with multilevel $p$-refinement in space in addition to multiple levels of $h$-refinement and/or algebraic multigrid. In UNIC, to partially address the memory issue, we employed parallelization by angle rather than $p$-refinement or $h$-refinement. Thus for the
SN2ND solver with a block diagonal connection in angle, it is not necessary to invest time defining a multigrid operation in angle because the method is immediately scalable in angle. It is expected that by incorporating the methodology that other codes have been developed to apply the CG method on up to 10,000 processors, the SN2ND solver can efficiently use over $1,000,000$ processors to solve a problem with 100 discrete ordinate directions.

The within-group equations given in Eq. (16) are coupled through the source $S_{g}$ and form a multigroup eigenvalue or fixed-source problem. The resulting multigroup equation is solved using a standard fission source iteration algorithm. To find the dominant eigenvalue, the power method has been used with a Tchebychev acceleration scheme. For a known fission source from the previous power iteration, another level of iteration on the multigroup scattering source is introduced because of the energy-angle coupling that occurs in the scattering operator. To decouple the group equations, a Gauss-Seidel procedure in energy is typically employed. The ultimate intent with UNIC is to implement a more general Krylov method where the current Gauss-Seidel scheme would likely serve as some part of a preconditioner where scalability in energy for timedependent problems is assumed. At this stage, we have Eq. (16) with a known out-of-group source from the most recent fission and multigroup scattering source iterations. To solve Eq. (16) in a parallel environment, the parallel CG implementation in PETSc [28] is used, where either the incomplete Cholesky (ICC) or symmetric successive overrelaxation (SSOR) preconditioner provided by PETSc was used. Each of these levels of iteration has simple dynamic error controls to minimize effort early in the calculation.

As mentioned above, SN2ND uses a conventional iterative scheme on the within-group scattering source (source or scattering iteration). Within each scattering iteration, the entire space-angle system needs to be solved using a CG operation because reflective boundary conditions inherently couple the individual angular systems together as mentioned earlier. Finally, the CG preconditioner for each discrete ordinate direction uses a preconditioned CG operation on the full spatial domain. Thus, the solution methodology in SN2ND consists of the following layers of iteration:

Fission source (inverse power) iteration for the eigenvalue (or fixed source)

Gauss-Siedel iteration over energy groups

Scattering iterations for the within-group scattering system

Conjugate gradient over the whole space-angle system

PETSc preconditioned conjugate gradient for each discrete ordinate $]\rfloor]$

At the lowest level, a parallel SSOR-preconditioned CG methodology available in PETSc is used to solve the full spatial domain for a given angle and energy group. This 
requires distributed sparse matrix storage of each symmetric positive-definite matrix. A preconditioned CG iteration is currently used for the second-lowest level, but a multigrid preconditioner is being developed to reduce sparse matrix storage requirements $[29,30]$. As mentioned above, DSA is used at the third-lowest level to accelerate the withingroup scattering source iteration.

With regard to parallelization, we employ the generic decomposition of space, angle and energy shown in Fig. 4. In the figure, the notation " $S, A, G$ " represents parallelization into $S$ spatial segments, $A$ angular segments, and $G$ energy segments. The main focus is to limit the communication costs of the various source operations necessary in the within-group formulations that are fundamental to modern neutron transport solvers. In this approach, each MPI process sees four communicators: space, angle, group, and the global communicator. The advantage of this approach is that the group and angle communication do not overlap with respect to space, and thus communication in these two directions can be done simultaneously on the parallel machine. Parallel hierarchy proceeds as energy, angle, and space in descending order. For the spatial part, classic domain decomposition is used where weights are applied to the vertices of elements to balance the local work with the communication costs required to connect the domain. Figure 5 shows an example of spatial domain decomposition obtained with the MeTiS package [31]. No solver in UNIC currently allows decomposition of the energy domain, although UNIC itself has been built this way from the beginning. It is important to note that the current spaceangle scheme in SN2ND is already enough to saturate the available memory and processors of the largest supercomputers available to open science research. In the current SN2ND solver, any number of angles can be distributed on a given process and it has been found that two to three angles per process works best.

\subsection{MOCFE Solver}

The MOCFE solver is based upon the first-order method of characteristics (MOC) $[32,33]$ and treats both two- and three-dimensional geometries. The long-term development goal for MOCFE is to use this solver on explicit geometry problems for high-fidelity benchmark calculations. However, the computational performance of MOC is too slow to be applied for three-dimensional whole core problems, and thus the short-term goal of MOCFE is to improve the crosssection generation process. As an example, a whole core planar two-dimensional MOC capability is a focused development effort intended to improve the spectral transition of the flux as it exits the core and enters the blanket and reflector in global three-dimensional solutions.

The derivation of the MOC equations begins with the first-order transport equation given in Eq. (7). Basically, noting that the first term on the left hand side of Eq. (7) is a directional derivative of $\psi_{g}$ in the direction of $\Omega$, the

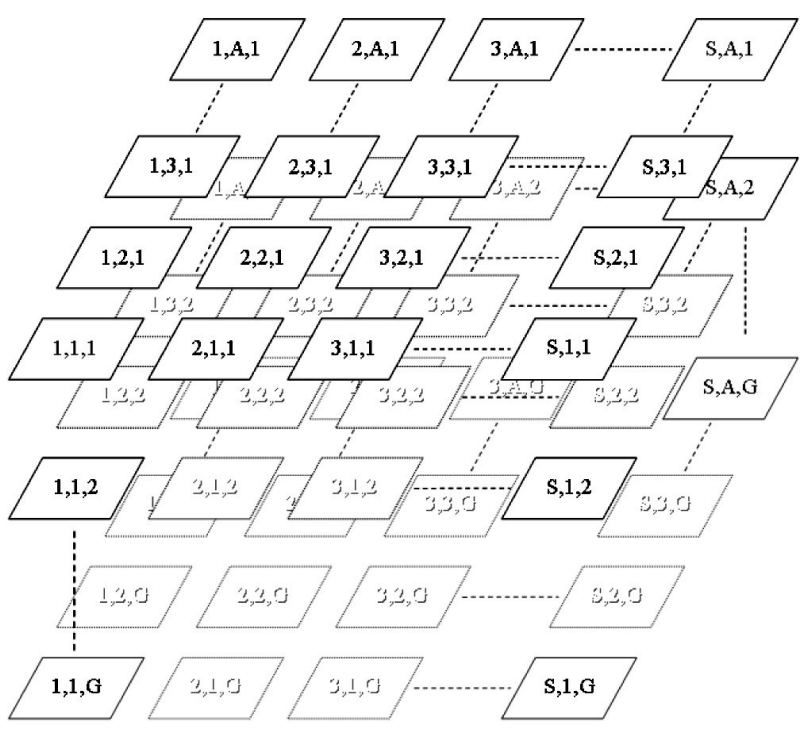

Fig. 4. Parallelization Strategy in UNIC
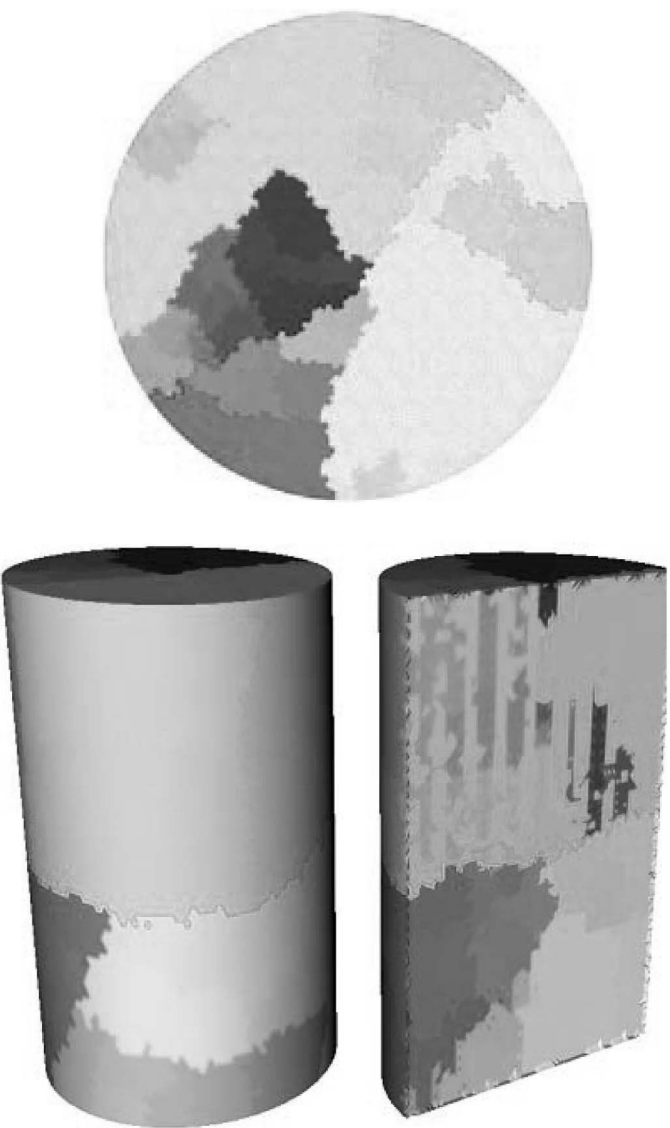

Fig. 5. An Example of MeTiS Decomposition for 512 Processors 
solution of Eq. (7) along a trajectory (ray) parallel to $\Omega$ (i.e., a characteristic line) can be obtained analytically for a given source distribution, assumed to be piecewise constant for this derivation. Thus, by evaluating the angular fluxes along the path of trajectories that are parallel to each direction in a selected angular cubature (i.e., ray tracing), the angular and scalar fluxes throughout the geometry on a finite element grid can be determined. This leads to a discrete form of the balance equation, which can be iteratively solved by updating the scattering and fission source terms with the most recent flux solution. The details of the MOC formulation were discussed previously [34,35] and thus not reproduced here.

Unlike most MOC solvers, MOCFE is based on a finite element discretization of the spatial domain rather than a combinatorial geometry. This is done primarily to handle truly arbitrary geometries that can account for structural deformation feedback, which is not easy to achieve with typical combinatorial geometry approaches. It is important to note that for a given combinatorial geometry, one can easily construct a finite element mesh, and thus the common simplified geometrical reactor core descriptions can be handled by the same rules under which existing MOC solvers operate. The current MOCFE solver can assume all finite elements supported by UNIC are cast onto the five primary ray tracing finite elements shown in Fig. 6. For two-dimensional elements, a standard line-intersection algorithm is used, while a ray-triangle intersection algorithm [36] is used for three-dimensional elements. This latter algorithm requires breaking the surfaces of the finite elements into triangles, the minimal decomposition of which is shown for each element type in Fig. 6. A scalable back projection algorithm has been constructed to define the trajectories in such a way that every element in the domain has crossing trajectories.
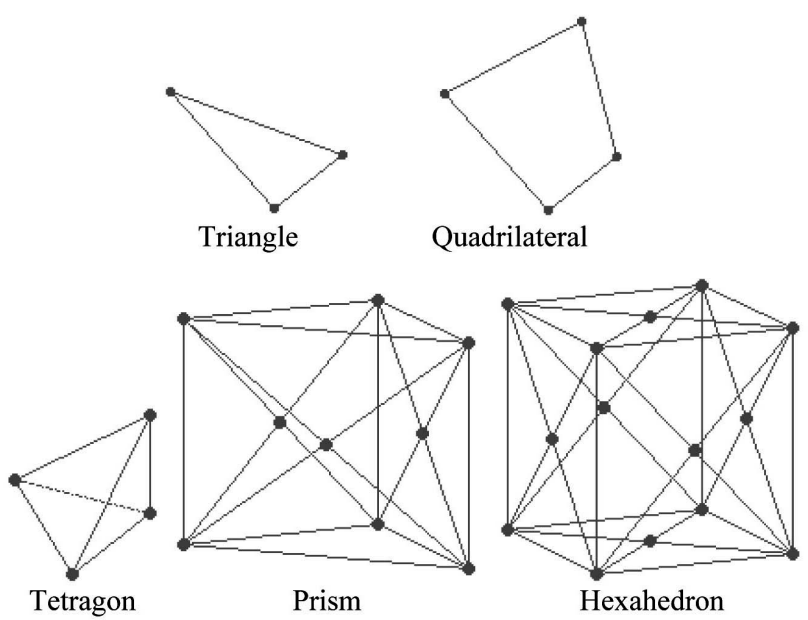

Fig. 6. Lowest-order Ray Tracing Elements in the MOCFE Solver
In the original development [34], parallel versions of MOCFE in two- and three-dimensional geometries were created assuming long characteristics over the whole domain. The trajectories were partitioned for each direction, but it was assumed that all processors have access to the full spatial domain. While this approach worked on a few hundreds of processors, the parallel algorithm employed was not scalable on petascale machines. After gaining some experience in the field, it was concluded that domain decomposition with per process segmentation of the characteristic lines is essential to have a scalable algorithm on petascale machines.

A new solver methodology has been developed using a hierarchical GMRES solver of PETSc [28] over the entire space-angle-energy system and thus a parallel decomposition in space-angle-energy. In angle, a straightforward partitioning of the discrete ordinates angles is used in a manner similar to that done in SN2ND. In space, standard spatial decomposition is used, which translates to the splitting or segmentation of the trajectories. Figure 7 shows an example of domain decomposition and trajectory segmentation for a two-dimensional assembly. The left hand picture shows a fuel assembly mesh decomposed into six pieces where the colors indicate geometric heterogeneity. In the left hand picture, the lower right image only applies coloring to the elements assigned to each processor and the upper image shows an uncolored mesh matching the right hand picture. The right hand picture shows the six-processor decomposed mesh overlaid with the intersecting trajectories (parallel lines). The breaking of each trajectory along the domain surface is clearly identifiable and reentrant trajectories can be identified on all six domains.

Along the border of each processor domain, the incoming and outgoing flux solution for each broken trajectory must be stored and communicated to the connected processor(s). One typically would consider the incoming flux to be a boundary condition for any given processor while the outgoing flux would be the boundary condition for the downwind processors. Because each domain can be reentrant with any given trajectory and any given trajectory can be reentrant on multiple domains with domain decomposition, the typical approach to sequenced sweeping is not wise. In MOCFE, the linear system of equations is recast into a form compatible with a Krylov subspace solver such that the outgoing angular trajectory flux on each domain is part of the Krylov vector space. This approach entails certain memory and computational costs and is currently being studied for use as a possible petascale algorithm for MOC. Note that MOCFE can incorporate the conventional sweep methodology, but that is not the focus of the development research.

The new version of MOCFE has been executed on 65,536 cores of BlueGene/P [37] for a mesh with 2.5 million elements. The observed strong and weak scaling 

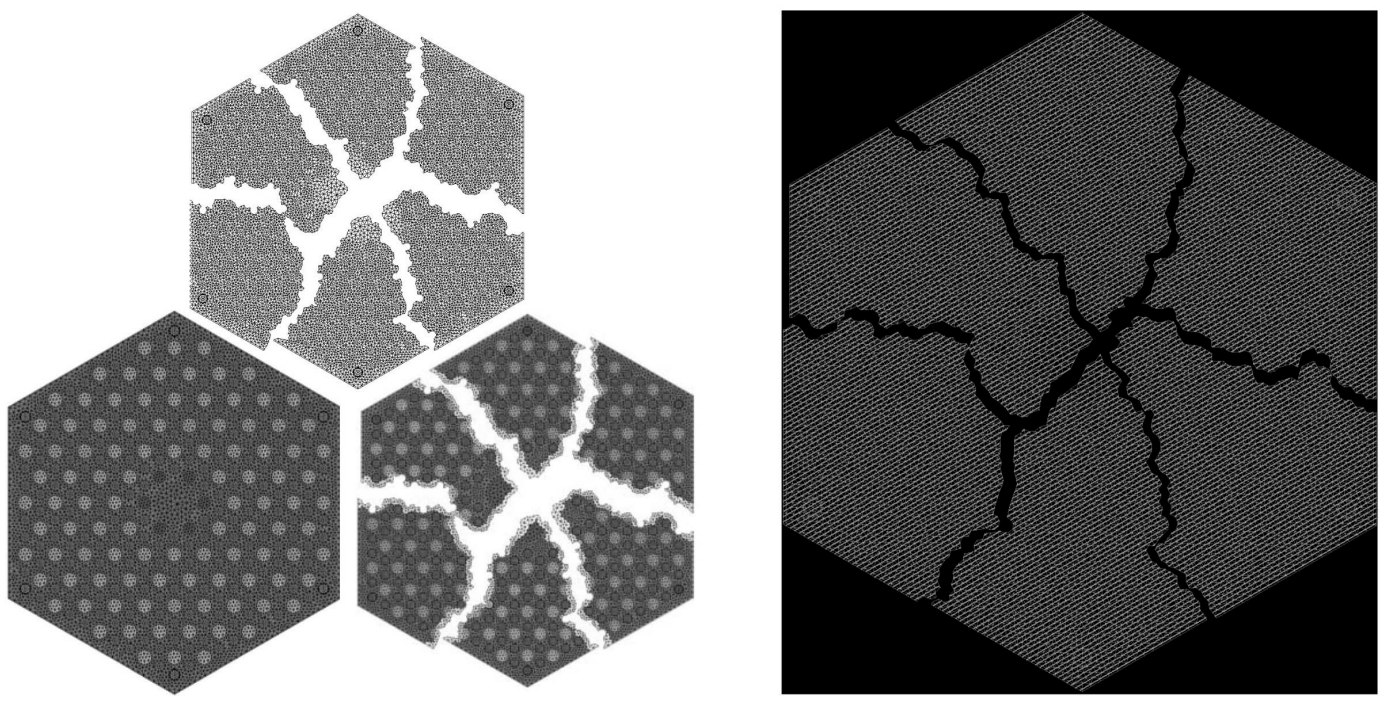

Fig. 7. Examples of Mesh Decomposition and Segmentation of Crossing Trajectories

is relatively poor primarily due to load balancing issues caused by a different number of characteristic lines passing through each subdomain. This load imbalance is directly attributable to the geometric heterogeneity of the domain (fuel assemblies have more geometry than shield assemblies) and thus one can observe a strong variation in the number of characteristic lines crossing any given domain with respect to angle. Two possible ways to fix the load imbalance are being examined at this point: one is to increase the number of angles per process (maximize the azimuthal angle between the directions) and the other is to increase the number of domains per process. Research into the first option is nearly complete with the general conclusion that a minimum load imbalance of 6.0 is achievable with low-order cubatures (20-50 directions), but this is unacceptable because it strongly restricts scalability $(<40 \%)$. The second option is likely better since the subdomains can be assigned based upon a combination of mesh density, element count, and geometric location. In the end, it is expected that a combination of the two will be necessary to make the load imbalance under 2.0, which is essential to obtain a scalability above $60 \%$.

The most important research that needs to be carried out for MOCFE is determining a better preconditioner on the space-angle system along with one on the entire spaceangle-energy system. As mentioned, the new MOCFE solver incorporates Krylov, specifically GMRES, on the entire space-angle system for which a diffusion synthetic acceleration system derived from the algebraic collapsing algorithm (ACA) is used as a preconditioner. This preconditioner is very cheap with respect to memory, but that also appears to severely restrict its accuracy. In this regard, a low-order $\mathrm{P}_{\mathrm{N}}$ system similar to the PN2ND solver will be investigated.

As a final note, this version of MOCFE is applicable to small-scale parallel machines (100s of processors) with parallelization in angle only. Most discrete ordinates solvers have good strong $(>50 \%)$ and weak $(>70 \%)$ scaling in angle. This should make the lattice calculations typically used in cross-section generation feasible using the existing solver scheme.

\subsection{Multigroup Cross Section Generation}

The generation of multigroup cross sections from evaluated nuclear data files remains one of the fundamental problems in reactor physics because of the need for accurate treatment of resonance effects. The methods used for crosssection processing must take into account both the resonance characteristics of intermediate nuclides (sodium, oxygen in the case of oxide fuel, and structural materials like iron, chromium, nickel, etc.) and the self-shielding in fissile and fertile nuclides. The rapidly varying elastic scattering cross sections of intermediate nuclides give rise to the fine structure of a typical fast reactor spectrum and require detailed treatment. The overlapping effect resulting from neighboring resonances either from the same nuclide or from different nuclides is another challenge in fast reactor analysis. Inelastic scattering also plays an important role in fast reactors, as it is the major cause of neutron slowingdown at high energies. Resonance shielding is generally treated using the Bondarenko self-shielding factor method [38-40] and the subgroup method [41-45], these being parameterizations of the resonance structure from which 
effective cross sections can be calculated for particular compositions and temperatures. An alternative approach developed at Argonne National Laboratory (ANL) is based on detailed spectrum calculations for specific compositions and temperatures [16] by representing all the resonance structures explicitly. At present, this approach is the most widely used in the United States of America for fast reactor modeling.

In the ETOE-2/MC ${ }^{2}-2 / \mathrm{SDX}$ code system [16] developed by ANL, the ETOE- 2 code processes the ENDF/B data and prepares the $\mathrm{MC}^{2}-2$ libraries, and the $\mathrm{MC}^{2}-2$ and SDX codes perform spectrum calculations and generate group constants for given compositions and temperatures. The current ultrafine group (UFG) energy structure chosen to form the $\mathrm{MC}^{2}-2$ libraries for smooth tabulated non-resonant data is 2082 groups with constant lethargy from $14.19 \mathrm{MeV}$ to $0.4 \mathrm{eV}$, but the ETOE-2 code allows any number of UFG lethargy intervals. Wide resonances are screened out and added to UFG smooth cross sections. The resonance data provided in the Reich-Moore formalism are converted into the multipole formalism [46] that preserves the general features required by the traditional resonance integral concept and the Doppler-broadening algorithm in the $\mathrm{MC}^{2}-2$ code without compromising rigor.

The $\mathrm{MC}^{2}-2$ code solves the slowing-down equation for specific compositions and temperatures with explicit representation of resonances. It solves the extended transport $\mathrm{P}_{1}$, extended transport $\mathrm{B}_{1}$, consistent $\mathrm{P}_{1}$, and consistent $\mathrm{B}_{1}$ fundamental mode UFG equations and accommodates highorder anisotropic scattering representations. Resolved and unresolved resonances are treated explicitly by the generalized resonance integral formulation based on the narrow resonance (NR) approximation including overlapping and Doppler broadening effects [47]. A fundamental mode homogeneous unit cell calculation is performed by solving the multigroup slowing-down equation above the resolved resonance energy and the continuous slowing-down equation below this range [48]. Equivalence theory is used to treat the heterogeneity effect, and isotropic approximation is used for fission, inelastic, and $(n, 2 n)$ sources. An alternative hyperfine group (HFG) integral transport calculation (RABANL) is available as an option to model the resolved resonances more accurately in the low-energy ranges where the narrow resonance approximation is not valid. Under this HFG method, every resolved resonance is represented by multiple points, which allows the neutron spectrum within a HFG to be approximated as a constant. RABANL performs a homogeneous or heterogeneous (pin or slab) unit cell calculation over the resonance region using the isotropic source approximation.

The SDX code is intended to supplement what is lacking in $\mathrm{MC}^{2}-2$ for treating the detailed heterogeneity effects associated with a complex reactor system. For each heterogeneous unit cell, heterogeneous resonance cross sections are computed in an intermediate group level of
230 groups for selected isotopes in the specified plate or pin types using equivalence theory or the rigorous RABANL heterogeneous treatment. These resonance cross sections are combined with the intermediate-group library data constructed from $\mathrm{MC}^{2}-2$ ultrafine group calculations, and then an infinite slab or cylinder integral transport calculation is performed in the intermediate group level for the unit cell. The integral transport calculation is based on a modified version of the CALHET code that applies the collision probability methods developed for RABANL. Spatial self-shielding factors and cell-averaged group cross sections are calculated.

In recent studies with the ENDF/B-VII.0 data [49,50], $\mathrm{MC}^{2}-2$ revealed some discrepancies. For example, the increased importance of resolved resonances in the ENDF/B-VII.0 data due to the extended upper energy cutoff and significantly increased number of resolved resonances required the use of RABANL for rigorous treatment of resolved resonances. However, its use is limited to the relatively low energy range where the isotropic source approximation is valid. Therefore, in order to generate the more accurate multigroup cross sections required for high-fidelity simulation with UNIC, $\mathrm{MC}^{2}-3$ has been developed by integrating the capabilities of $\mathrm{MC}^{2}-2$ and SDX and by eliminating various limitations and approximations of the current methods as discussed below.

The generalized resonance integral method of $\mathrm{MC}^{2}-2$ for calculating self-shielded UFG cross sections is based on the analytical integration of resonances over the entire energy range rather than each UFG interval. The approximate integration interval introduces the so-called resonance tail effect, and thus wide resonances are screened out and added to UFG smooth cross sections. The resonance tail effect becomes more pronounced as the number of energy groups in the target library increases, and thus the analytic resonance integral method becomes less attractive for the high-fidelity simulation with UNIC where the number of energy groups is expected to increase with increases in computing power. Therefore, a new approach based on the numerical integration of pointwise cross sections with the narrow resonance approximation has been introduced in $\mathrm{MC}^{2}-3$. Doppler broadened pointwise cross sections are reconstructed within the code using the resonance parameters. The NR fluxes are obtained using the pointwise total cross sections of a specified composition. The self-shielded UFG cross sections are determined using numerical integration over the exact energy interval of each UFG as:

$$
\bar{\sigma}_{x}^{i}=\int_{\Delta u} \frac{\sigma_{x}^{i}(u)}{\Sigma_{t}(u)} d u / \int_{\Delta u} \frac{1}{\Sigma_{t}(u)} d u
$$

where $\sigma_{x}^{i}(u)$ is the pointwise cross section of reaction type $x$ for isotope $i$ in the lethargy interval $\Delta u$ and $\Sigma_{t}(u)$ is 
the macroscopic total cross section of the composition. In a similar way, the $l$-th order self-shielded cross sections are calculated by weighting with the $l$-th order flux moment approximated as $1 /\left[\Sigma_{t}(u)\right]^{l+1}$.

The continuous slowing-down method of $\mathrm{MC}^{2}-2$ used for the UFG spectrum calculation in the resolved resonance energy range becomes more approximate when light elements (especially hydrogen) are included. In order to minimize this approximate nature and to simplify the program structure, the continuous slowing-down method has been replaced with the multigroup method. Consequently, the UFG spectrum is calculated by solving the multigroup $\mathrm{P}_{1}$ equations for the entire energy range with an extended transport approximation $[15,17]$ up to $\mathrm{P}_{9}$. The isotropic approximation of inelastic scattering and $(\mathrm{n}, 2 \mathrm{n})$ reactions has also been removed by introducing anisotropic scattering matrices. In addition, the isotopic fission spectra depending on incident neutron energy are available, and the resonance-like cross sections of the intermediate mass nuclides such as iron, nickel, and chromium above the resonance energy range are selfshielded using the NR approximation.

For the HFG spectrum calculation in the resolved resonance range, the isotropic source approximation is eliminated by incorporating anisotropic scattering sources. This allows increasing the upper energy limit of HFG calculation up to the top of the resolved resonance region of intermediate mass nuclides $(\sim 1 \mathrm{MeV})$ where the isotropic source approximation is not valid. As mentioned above, the HFG structure is constructed in such a way that the neutron spectrum within a HFG can be approximated to be constant. Thus, if $N$ is the scattering order in the centerof-mass system (CMS) given in the ENDF/B data, the scattering transfer cross section of order $l$ from the source group $g$ to the sink group $g^{\prime}$ in the laboratory system (LS) is calculated using

$$
\begin{gathered}
\sigma_{s l}\left(g \rightarrow g^{\prime}\right) \cong \int_{u_{g^{\prime}-1}}^{u_{u_{s}^{*}}^{*}} d u^{\prime} \int_{u_{g-1}^{u_{g}}}^{u_{g}} d u \frac{\sigma_{s}(u) e^{-\left(u^{\prime}-u\right)} P_{l}\left[\mu_{0}\left(u, u^{\prime}\right)\right]}{(1-\alpha)} \\
\sum_{n=1}^{N}(2 n+1) f_{n}(u) P_{n}\left[\mu_{c}\left(u, u^{\prime}\right)\right]
\end{gathered}
$$

where $f_{n}$ is the $n$-th order Legendre expansion coefficient in CMS, $u_{\mathrm{g}^{\prime}}^{*}$ and $u_{\mathrm{g}-1}^{*}$ are the reachable energy boundaries, and $\alpha=(A-1)^{2} /(A+1)^{2}$ with $A$ being the atomic mass. The cosines of the scattering angles in LS and CMS are given by

$$
\mu_{c}\left(u, u^{\prime}\right)=\frac{1}{1-\alpha}\left[2 e^{-\left(u^{\prime}-u\right)}-(1+\alpha)\right]
$$

$$
\mu_{s}\left(u, u^{\prime}\right)=\frac{1}{2}\left[(A+1) e^{-\left(u^{\prime}-u\right) / 2}-(A-1) e^{-\left(u-u^{\prime}\right) / 2}\right]
$$

The heterogeneous cell calculation capability of SDX for slab and cylindrical unit cells has also been integrated into $\mathrm{MC}^{2}-3$ by extending the spatial transport calculation from an intermediate group level to the ultrafine group level. Either the collision probability method (CPM) or the method of characteristics (MOC) can be used. The UFG cross sections of each region are determined using the equivalence theory. That is, the UFG cross sections are self shielded using the composition-dependent potential scattering cross section, augmented by the geometrydependent escape cross section. Region-wise or cellaverage broad-group cross sections are produced. A HFG transport calculation capability with an anisotropic scattering source representation is currently being implemented. The $\mathrm{MC}^{2}-3$ code is also being built to use the MOCFE solver of UNIC so that the approximations imposed by the simplified one-dimensional cell calculations are reduced and the material and temperature distributions that appear in whole-core transport calculations are correctly incorporated.

\section{TEST RESULTS}

To test the performance of $\mathrm{MC}^{2}-3$ and UNIC, we analyzed several benchmark problems and fast critical experiments. The experiments analyzed include eight critical assemblies [51] of Los Alamos National Laboratory (LANL), ZPR-6 Assemblies 6A (ZPR-6/6A) and 7 (ZPR6/7) [51,52], three loadings of ZPPR-15A [53], six phases of ZPPR-21 [51], and three BFS assemblies [52,54]. Using the ENDF/B-VII.0 data [55], isotopic multigroup cross sections were generated for each composition using $\mathrm{MC}^{2}-3$. The $\mathrm{MC}^{2}-3$ and UNIC results were compared with the reference Monte Carlo solutions obtained with MCNP5 [56] or VIM [57]. For detailed as-built models, the results were also compared against the experimental results.

The eight LANL critical assemblies include Godiva, Bigten, Flattop-25, Flattop-Pu, Flattop-23, Jezebel, Jezebel$\mathrm{Pu}$, Jezebel-23, three of which-Flattop-Pu, Jezebel, and Jezebel-Pu - contain plutonium fuel. The core regions of ZPR-6/6A and ZPR-6/7 are composed of uranium and plutonium fuels, respectively. Of the six phases of ZPPR21 , the first phase, ZPPR-21A, contains only plutonium fuel to take advantage of the inherent neutron source of $\mathrm{Pu}-240$. The enriched uranium is then progressively substituted for plutonium in Phases B through E so that the final phase, ZPPR-21F, has only uranium fuel loading, which requires an external neutron source to reach criticality. Three loading configurations of the ZPPR-15A 
experiments tested in this study are the initial criticality configuration (loading 15), a reference configuration for sodium void worth measurement (loading 16), and a configuration with an 18-inch sodium void in part of the inner core (loading 20). Among the three BFS assemblies, the BFS-75-1 assembly consists of two enriched uranium fuel zones and two composite radial blankets (U-238 metal and $\mathrm{UO}_{2}$ ), while BFS-73-1 has only a single enriched uranium fuel zone and one radial blanket $\left(\mathrm{UO}_{2}\right)$. The difference in composition between the two assemblies is that zirconium metal disks are included in the unit fuel cell of BFS-75-1 but not in that of BFS-73-1. A unit fuel cell of the BFS-75-1 critical assembly is more heterogeneous in configuration than that of the BFS-73-1 since a highly enriched uranium disk is placed between medium enriched metal uranium disks. Similar to BFS-73-1, the BFS-55-1 assembly has a single enriched uranium fuel zone and one radial blanket, containing plutonium disks in the unit fuel cell unlike the other two assemblies.

\subsection{Homogeneous Medium and Unit Cell Calculations}

Using the improved methods of $\mathrm{MC}^{2}-3$, the previously observed discrepancies of $\mathrm{MC}^{2}-2$ with the ENDF/B-VII.0 data and with the increased number of broad groups were first examined by performing homogeneous medium calculations. The neuron spectra were calculated by solving three different slowing-down equations: the UFG equation with anisotropic scattering sources, the HFG equation with isotropic (in CMS) sources as in RABANL, and the HFG equation with anisotropic sources. For the UFG calculation, the self-shielded cross sections were obtained by the numerical integration of pointwise cross sections with the narrow resonance approximation as discussed earlier. Figure 8 compares the UFG spectra obtained from the three slowing-down calculations for the inner core composition of ZPR-6/6A. The HFG calculations were applied to the energy range below $1 \mathrm{MeV}$ because the highest resolved resonance energy of Cr-52 included in the composition is $980 \mathrm{keV}$ in the ENDF/B-VII.0 data. As can be seen, the UFG spectrum agrees well with the reference HFG spectrum obtained with an anisotropic source. This result indicates that the numerical integration of pointwise cross sections with the narrow resonance approximation is sufficiently accurate for self-shielding the UFG cross sections. Thus, it can be concluded that the required use of RABANL in $\mathrm{MC}^{2}-2$ calculations with the ENDF/B-VII.0 data is largely due to the increased resonance tail effects of the analytic resonance integral method. This conclusion is also supported by the observation that without the RABANL hyperfine group calculation, $\mathrm{MC}^{2}-2$ produces large errors in UFG total cross sections even at the infinite-dilute condition, whereas $\mathrm{MC}^{2}-3$ generates accurate infinite-dilute UFG cross sections.

It can also be seen from Fig. 8 that the HFG spectrum obtained with isotropic (in CMS) sources is noticeably different from the reference spectrum above $100 \mathrm{keV}$ because of the increased importance of anisotropic scattering due to $\mathrm{p}$-, $\mathrm{d}$-, and f-wave scattering. This result suggests that in this energy range, anisotropic scattering in the CMS should be accounted for. As expected, it was also observed that for low-energy groups around a relatively wide actinide resonance that spans several ultrafine groups, the UFG cross sections obtained with the NR approximation show non-negligible differences from those collapsed with the HFG flux solution. As an example, Fig. 9 compares the NR approximation flux superimposed on the UFG flux with the HFG flux around a U-238 resonance at $189.68 \mathrm{eV}$. A noticeable difference can be seen below the resonance peak. As a result, for the UFG between 186.2 to $186.7 \mathrm{eV}$, the total cross section based on the NR approximation shows $\sim 5 \%$ deviation from that obtained with the HFG solution. As can be seen in Fig. 8, the neutron flux in this low energy range is generally very low in fast reactors, and thus the impacts of the UFG cross section errors are negligible. However, this epithermal energy range could be important for certain applications such as the steamflooded, gas-cooled fast reactor analysis.

Table 1 compares the infinite multiplication factors obtained with $\mathrm{MC}^{2}-3$ with MCNP5 or VIM Monte Carlo solutions for the various homogenized compositions and unit cells of the ZPR-6/6A, ZPR-6/7, ZPPR-15A, and ZPPR21 assemblies. Based on the aforementioned observations, the default UFG slowing-down option was used for $\mathrm{MC}^{2}-3$ calculations. The ZPR- 6 and ZPPR fast critical assemblies are split-table machines holding lattices of stainless steel tubes with a square cross section of $5.5 \mathrm{~cm}$ outside dimension. These tubes are loaded with stainless steel drawers filled with plate-type unit cell loadings, as depicted

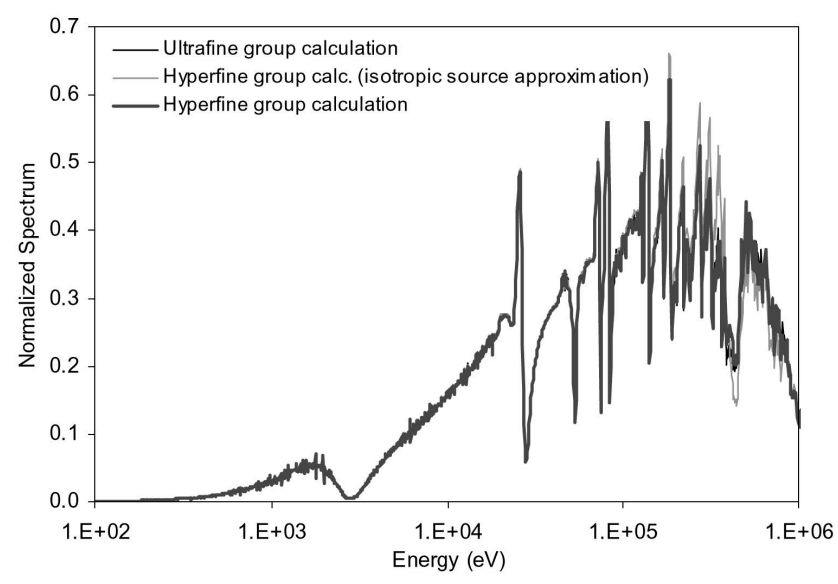

Fig. 8. Comparison of UFG Spectra Obtained with Three Different Slowing-Down Calculations 


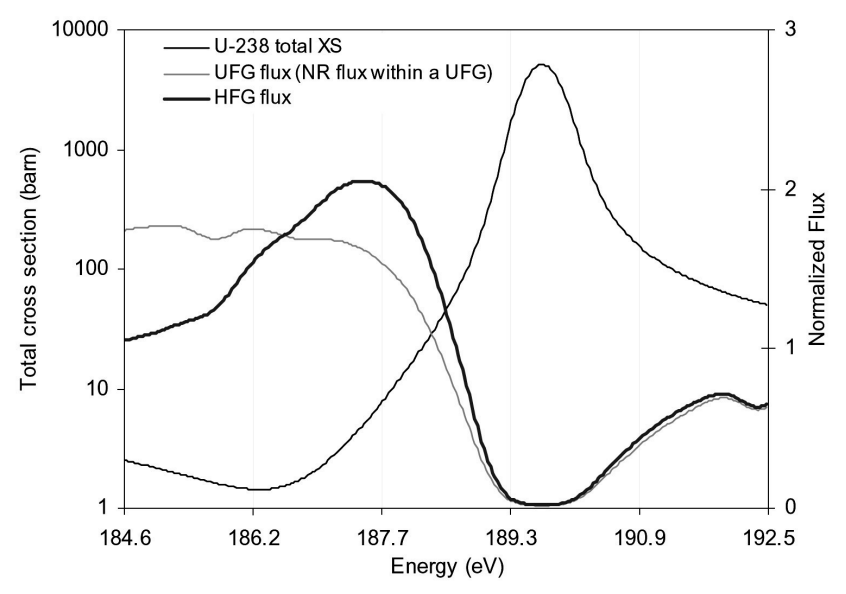

Fig.9. Comparison of NR Approximation Fluxes Superimposed on UFG Flux and HFG Flux around U-238 Resonance at $189.68 \mathrm{eV}$ in Fig. 10 for the loading 106 of ZPR-6/7. The unit cell loadings are approximately one-dimensional (1-D), but the variations in plate dimensions make them threedimensional (3-D) as shown in Fig. 11. Since the $\mathrm{MC}^{2}-3$ unit cell calculation is based on 1-D slab geometries, the 3-D matrix tube loading was transformed to an equivalent 1-D computational model by smearing the structural materials from the periphery of the physical cell into the regions that do not include heavy elements. It can be seen that the infinite multiplication factors of $\mathrm{MC}^{2}-3$ agree well with the Monte Carlo solutions, but $\mathrm{MC}^{2}-3$ consistently underestimates the infinite multiplication factor compared to MCNP5 or VIM. It is also noted that the local heterogeneity effect is very large for ZPR drawers (e.g., $3,744 \mathrm{pcm}$ for the ZPR-6/7 inner core drawer, $1,829 \mathrm{pcm}$ for the ZPPR-15A inner core drawer, and $1,009 \mathrm{pcm}$ for the ZPPR-15A outer core drawer), and this large local heterogeneity effect is accurately accounted for in the 1$\mathrm{D}$ cell model of $\mathrm{MC}^{2}-3$.

Table 1. Infinite Multiplication Factors of Homogenized Compositions and Unit Cells

\begin{tabular}{|c|c|c|c|c|}
\hline Model & Assembly & Drawer & Monte Carlo & $M C^{2}-3^{\text {a) }}$ \\
\hline \multirow{16}{*}{$\begin{array}{l}\text { Homogenized } \\
\text { Composition }\end{array}$} & \multirow{4}{*}{ ZPR-6/6A } & Inner core & $1.22945 \pm 0.00038$ & 7 \\
\hline & & Outer core & $1.22482 \pm 0.00048$ & -31 \\
\hline & & Radial blanket & $0.33513 \pm 0.00043$ & -59 \\
\hline & & Axial blanket & $0.33215 \pm 0.00048$ & -22 \\
\hline & \multirow{4}{*}{ ZPR-6/7 } & Inner core & $1.24366 \pm 0.00046$ & -87 \\
\hline & & Outer core & $1.23802 \pm 0.00047$ & -128 \\
\hline & & Radial blanket & $0.33445 \pm 0.00042$ & 10 \\
\hline & & Axial blanket & $0.32975 \pm 0.00043$ & -27 \\
\hline & \multirow{2}{*}{ ZPPR-15A } & Inner core & $1.17243 \pm 0.00018$ & -247 \\
\hline & & Outer core & $1.65167 \pm 0.00019$ & -284 \\
\hline & ZPPR-21A & Inner core & $2.49784 \pm 0.00013$ & -55 \\
\hline & ZPPR-21B & Inner core & $2.38138 \pm 0.00012$ & -77 \\
\hline & ZPPR-21C & Inner core & $2.27032 \pm 0.00012$ & -76 \\
\hline & ZPPR-21D & Inner core & $2.16489 \pm 0.00010$ & -104 \\
\hline & ZPPR-21E & Inner core & $2.08656 \pm 0.00011$ & -108 \\
\hline & ZPPR-21F & Inner core & $2.04243 \pm 0.00011$ & -121 \\
\hline \multirow{3}{*}{ 1-D Unit Cell } & ZPR-6/7 & Inner core & $1.28110 \pm 0.00028$ & 286 \\
\hline & \multirow{2}{*}{ ZPPR-15A } & Inner core & $1.19072 \pm 0.00021$ & -54 \\
\hline & & Outer core & $1.66176 \pm 0.00021$ & -29 \\
\hline
\end{tabular}

\footnotetext{
a) Difference from Monte Carlo solution in $\Delta \mathrm{k} \mathrm{pcm}$
} 


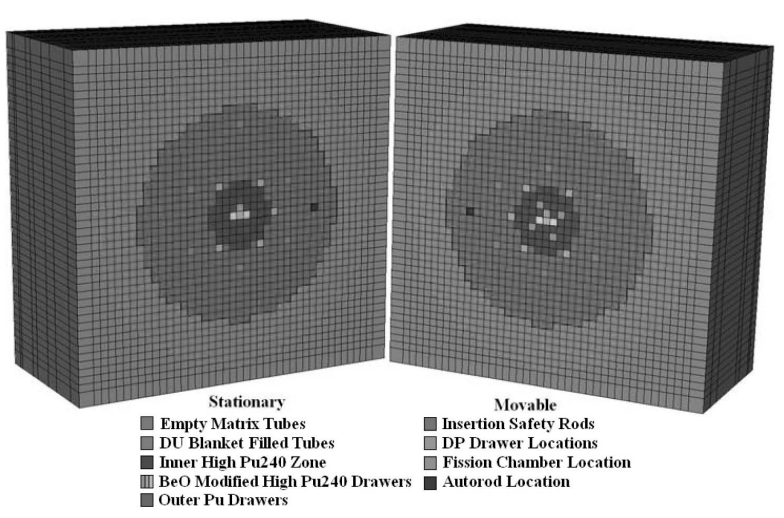

Fig. 10. Schematics of ZPR-6/7 Loading 106

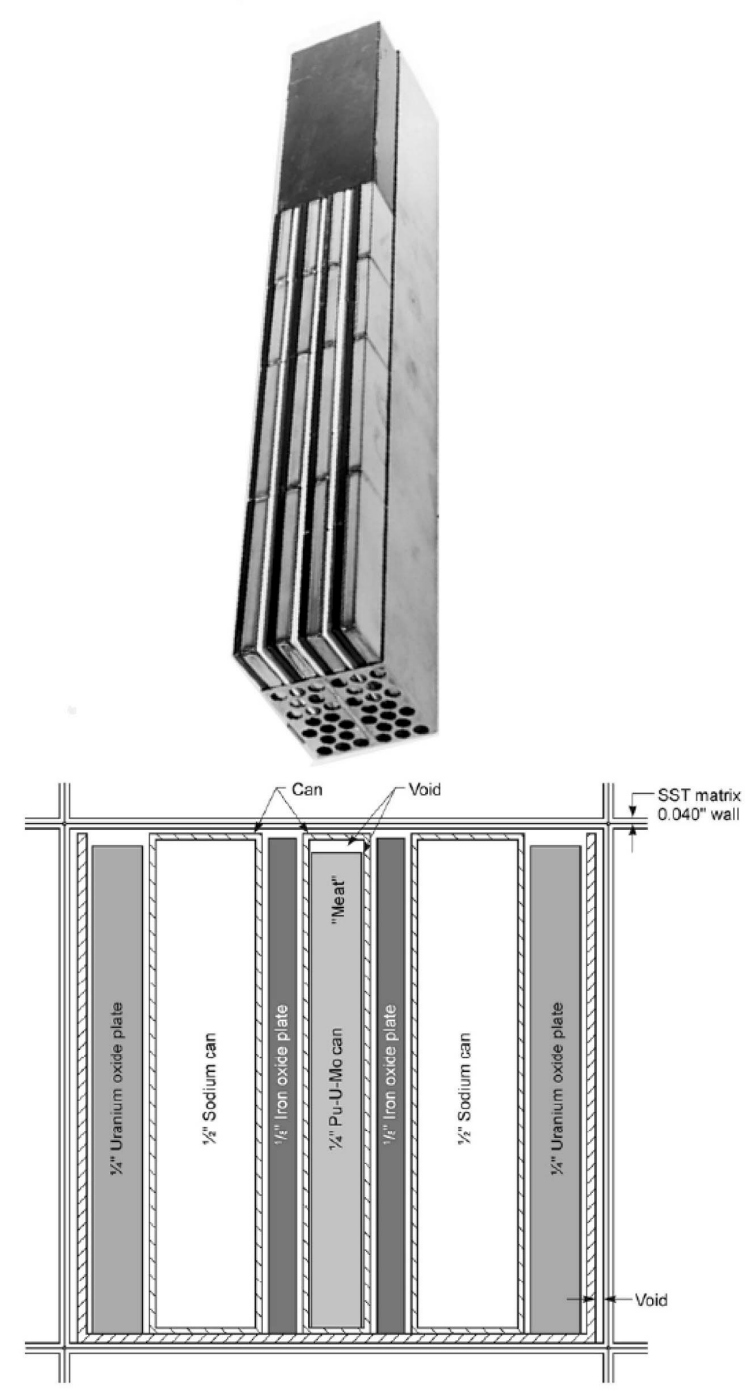

Fig. 11. Typical ZPR Drawer (top) and Cross Section of Core Unit Cell Showing Matrix and Plate-loaded Drawer of ZPR6/7 (bottom)

\subsection{Simplified Benchmark Problems}

As a next step to test the performance of $\mathrm{MC}^{2}-3$, the simplified benchmark problems for the experiments discussed above were analyzed. The benchmark models in spherical or cylindrical (R-Z) geometries with homogenized compositions were used. For each distinct composition, 230-group cross sections were generated with $\mathrm{MC}^{2}-3$ using the default UFG slowing-down calculation option. The core calculations were performed using the TWODANT discrete ordinate code [27] with $\mathrm{S}_{24}$ angular quadrature and $\mathrm{P}_{3}$ or $\mathrm{P}_{5}$ anisotropic scattering order. Table 2 compares the effective multiplication factors obtained from $\mathrm{MC}^{2}-3 / \mathrm{TWODANT}$ calculations with MCNP5 or VIM Monte Carlo solutions. As can be seen, the $\mathrm{MC}^{2}-3 / \mathrm{TWODANT}$ results agree well with the Monte

Table 2. Effective Multiplication Factors of Simplified Benchmark Problems with Homogenized Compositions

\begin{tabular}{|c|c|c|}
\hline Assembly & Monte Carlo & $\begin{array}{c}\mathrm{MC}^{2}-3 / \\
\text { TWODANT }^{\text {a) }}\end{array}$ \\
\hline GODIVA & $0.99996 \pm 0.00032$ & 5 \\
\hline BIGTEN & $0.99513 \pm 0.00027$ & 187 \\
\hline FLATTOP-25 & $1.00212 \pm 0.00035$ & 101 \\
\hline FLATTOP-Pu & $1.00072 \pm 0.00034$ & -134 \\
\hline FLATTOP-23 & $0.99921 \pm 0.00034$ & -26 \\
\hline JEZEBEL & $1.00028 \pm 0.00030$ & -129 \\
\hline JEZEBEL-Pu & $0.99944 \pm 0.00031$ & -27 \\
\hline JEZEBEL-23 & $1.00007 \pm 0.00031$ & -14 \\
\hline ZPR-6/6A & $0.99609 \pm 0.00023$ & -15 \\
\hline ZPR-6/7 & $0.98671 \pm 0.00022$ & -18 \\
\hline ZPPR-15A L15 & $0.98386 \pm 0.00026$ & -23 \\
\hline ZPPR-15A L16 & $0.98081 \pm 0.00028$ & -11 \\
\hline ZPPR-15A L20 & $0.98413 \pm 0.00025$ & -46 \\
\hline ZPPR-21A & $0.99869 \pm 0.00020$ & -133 \\
\hline ZPPR-21B & $0.99293 \pm 0.00020$ & 70 \\
\hline ZPPR-21C & $0.99923 \pm 0.00018$ & -90 \\
\hline ZPPR-21D & $1.00345 \pm 0.00020$ & 141 \\
\hline ZPPR-21E & $1.00485 \pm 0.00020$ & 49 \\
\hline ZPPR-21F & $1.00612 \pm 0.00020$ & -123 \\
\hline BFS-55-1 & $0.97511 \pm 0.00016$ & 197 \\
\hline BFS-73-1 & $0.99553 \pm 0.00017$ & 149 \\
\hline BFS-75-1 & $0.99349 \pm 0.00017$ & 139 \\
\hline
\end{tabular}

a) Difference from Monte Carlo solution in $\Delta \mathrm{k} \mathrm{pcm}$ 
Carlo solutions: within $\sim 200 \mathrm{pcm}$ for the LANL critical assemblies and three BFS experiments; within $\sim 150 \mathrm{pcm}$ for six loadings of the ZPPR-21; within $\sim 50 \mathrm{pcm}$ for three loadings of ZPPR-15A; and within $\sim 20 \mathrm{pcm}$ for the ZPR$6 / 6 \mathrm{~A}$ and ZPR-6/7. No obvious trend is observed in the differences.

The differences from Monte Carlo solutions of the TWODANT multiplication factors obtained with $\mathrm{MC}^{2}-2$ and $\mathrm{MC}^{2}-3$ cross sections are also compared in Fig. 12. The figure shows that the $\mathrm{MC}^{2}-3$ cross sections improve the multiplication factors significantly. In particular, the solutions for LANL assemblies improved markedly, mainly due to the new capability of treating anisotropic inelastic scattering.

Fission reaction rates of major isotopes for six LANL critical assemblies were calculated at the center of the assemblies, and compared with the measured values and MCNP results. As shown in Table 3, the fission reaction rate ratios of U-233, Np-237, U-238, and Pu-239 to U-235 calculated with $\mathrm{MC}^{2}-3 / \mathrm{TWODANT}$ agree very well with those of MCNP, although all the calculated values with ENDF/B-VII.0 are smaller than the measurements by up to $\sim 4 \%$. These results indicate that the deviations from the measurements are mostly due to the ENDF/B-VII.0 data itself rather than the multigroup cross sections generation method of $\mathrm{MC}^{2}-3$.

\subsection{Three-Dimensional Homogenized Core Benchmarks}

The three solver modules of UNIC were first tested using three-dimensional homogenized benchmark problems with given sets of group cross sections. One such series

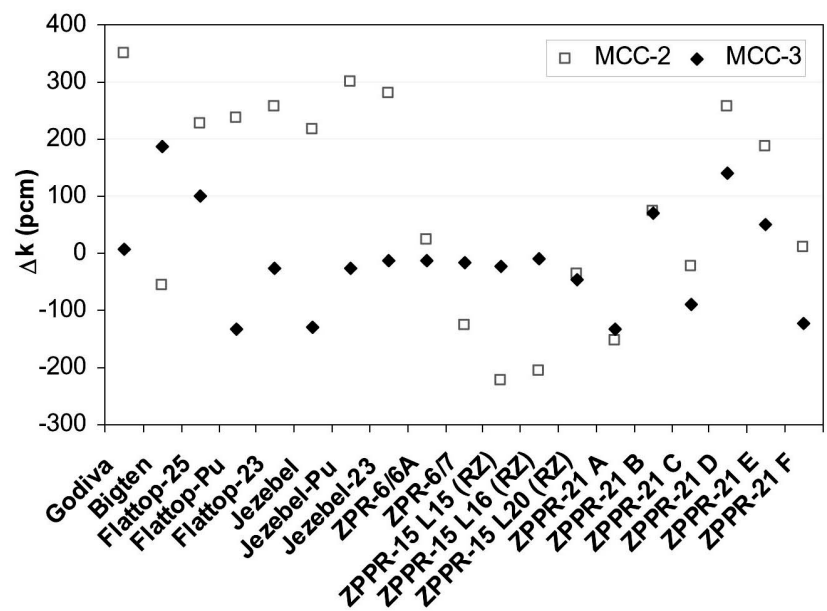

Fig. 12. Differences from Monte Carlo Solutions of TWODANT Multiplication Factors Obtained with $M^{2}-2$ and $\mathrm{MC}^{2}-3$ Cross Sections of benchmarks applicable to both thermal and fast reactors was proposed by Takeda [58]. The first three benchmarks are Cartesian, PWR-like thermal reactors while the fourth is a hexagonal configuration representative of a fast reactor. The original purpose of the benchmark was to compute the control rod worth. The first and fourth benchmarks were solved with UNIC. The first benchmark proposed is a small cubical reactor $50 \mathrm{~cm}$ on a side. The geometry has $1 / 4$ symmetry in the radial plane and $1 / 8$ symmetry overall. The fourth benchmark is a medium-sized fast reactor 105 $\mathrm{cm}$ tall with an assembly pitch of $12.99038 \mathrm{~cm}$ and a full 8ring geometry definition. The geometry has $1 / 12$ symmetry in the radial plane. For the first and fourth benchmarks, two- and four-group macroscopic cross sections were respectively provided for each region in the problem in addition to a reference Monte Carlo solution.

Two sets of meshes were used for PN2ND and SN2ND calculations. For benchmark 1, hexahedron meshes of 4,352 and 15,625 elements were used, resulting in 19,079 and 24,362 vertices, respectively. A tetrahedral mesh of 11,812 elements (19,079 vertices) and hexahedral mesh of 5,240 elements $(24,362$ vertices) were used for benchmark 4. MOCFE calculations were performed with linear tetrahedral meshes upwards of 496,979 and 3,448,832 elements for benchmarks 1 and 4, respectively. The angular approximation varied from $\mathrm{P}_{1}$ through $\mathrm{P}_{11}$ for $\mathrm{PN} 2 \mathrm{ND}$, and up to 288 discrete angles on the sphere were used for SN2ND and MOCFE. Tables 4 and 5 summarize the eigenvalue results for benchmark 1 and 4, respectively, showing the results of each solver in UNIC in addition to solutions from VARIANT. Although the eigenvalue results are somewhat scattered, the calculated control rod worths are within one standard deviation of the reference solutions, except for the full control rod worth of the fourth benchmark. The convergence behavior of the solutions indicated that more mesh refinement is necessary in all solvers of UNIC to converge to the reference solution. For example, Table 6 shows the eigenvalue solution of MOCFE for the mesh refinement study for the benchmark 4, where the angular cubature was fixed at 18 directions and a maximum trajectory area of $0.05 \mathrm{~cm}^{2}$ was used. An asymptotic convergence toward the reference solution is observable, but the last two meshes still result in nearly a 40 to 50 pcm change in the eigenvalue. It is also noted that MOCFE requires many more spatial elements than SN2ND for these homogenized problems, a typical by-product of the flat source approximation in MOC.

Assembly homogenized benchmark calculations were also performed for the Advanced Burner Test Reactor (ABTR) design [59,60]. Using the $\mathrm{MC}^{2}-3$ code, composition-dependent isotopic cross sections were generated in 9- and 33-group structures. The reference eigenvalue solutions obtained with 9- and 33-group MCNP calculations were $1.00813 \pm 0.00004$ and $1.01406 \pm$ 0.00004 , respectively. PN2ND and SN2ND calculations were performed with various angular approximations and 
Table 3. Comparison of Measured and Calculated Spectral Indices for LANL Assemblies

\begin{tabular}{|c|c|c|c|c|c|c|}
\hline Assembly & & Data & $\sigma_{f}^{U 238} / \sigma_{f}^{U 235}$ & $\sigma_{f}^{N p 237} / \sigma_{f}^{U 235}$ & $\sigma_{f}^{U 233} / \sigma_{f}^{U 235}$ & $\sigma_{f}^{P u 239} / \sigma_{f}^{U 235}$ \\
\hline \multirow{3}{*}{ GODIVA } & \multicolumn{2}{|c|}{ Experiment } & $\begin{array}{c}0.1643 \\
\pm 0.0018\end{array}$ & $\begin{array}{r}0.8516 \\
\pm 0.013\end{array}$ & $\begin{array}{c}1.59 \\
\pm 0.03\end{array}$ & $\begin{array}{r}1.4152 \\
\pm 0.0250\end{array}$ \\
\hline & \multirow{2}{*}{$\mathrm{C} / \mathrm{E}$} & MCNP & 0.960 & 0.975 & 0.987 & 0.977 \\
\hline & & $\mathrm{MC}^{2}-3$ & 0.958 & 0.974 & 0.987 & 0.977 \\
\hline \multirow{3}{*}{ JEZEBEL } & \multicolumn{2}{|c|}{ Experiment } & $\begin{array}{c}0.2133 \\
\pm 0.0023\end{array}$ & $\begin{array}{c}0.9835 \\
\pm 0.016\end{array}$ & $\begin{array}{c}1.578 \\
\pm 0.027\end{array}$ & $\begin{array}{c}1.4609 \\
\pm 0.013\end{array}$ \\
\hline & \multirow{2}{*}{$\mathrm{C} / \mathrm{E}$} & MCNP & 0.978 & 0.988 & 0.986 & 0.975 \\
\hline & & $\mathrm{MC}^{2}-3$ & 0.968 & 0.986 & 0.987 & 0.975 \\
\hline \multirow{3}{*}{ JEZEBEL -23 } & \multicolumn{2}{|c|}{ Experiment } & $\begin{array}{c}0.2131 \\
\pm 0.0026\end{array}$ & $\begin{array}{c}0.9970 \\
\pm 0.015\end{array}$ & & \\
\hline & \multirow{2}{*}{$\mathrm{C} / \mathrm{E}$} & MCNP & 0.989 & 0.984 & & \\
\hline & & $\mathrm{MC}^{2}-3$ & 0.988 & 0.998 & & \\
\hline \multirow{3}{*}{ FLATTOP-25 } & \multicolumn{2}{|c|}{ Experiment } & $\begin{array}{c}0.1492 \\
\pm 0.0016\end{array}$ & $\begin{array}{l}0.7804 \\
\pm 0.01\end{array}$ & $\begin{array}{c}1.608 \\
\pm 0.003\end{array}$ & $\begin{array}{c}1.3847 \\
\pm 0.012\end{array}$ \\
\hline & \multirow{2}{*}{$\mathrm{C} / \mathrm{E}$} & MCNP & 0.968 & 0.988 & 0.975 & 0.982 \\
\hline & & $\mathrm{MC}^{2}-3$ & 0.966 & 0.988 & 0.975 & 0.982 \\
\hline \multirow{3}{*}{ FLATTOP-Pu } & \multicolumn{2}{|c|}{ Experiment } & $\begin{array}{r}0.1799 \\
\pm 0.002 \\
\end{array}$ & $\begin{array}{c}0.8561 \\
\pm 0.012 \\
\end{array}$ & & \\
\hline & \multirow{2}{*}{$\mathrm{C} / \mathrm{E}$} & MCNP & 0.984 & 0.996 & & \\
\hline & & $\mathrm{MC}^{2}-3$ & 0.970 & 0.992 & & \\
\hline \multirow{3}{*}{ FLATTOP-23 } & \multicolumn{2}{|c|}{ Experiment } & $\begin{array}{c}0.1916 \\
\pm 0.0021\end{array}$ & $\begin{array}{c}0.9103 \\
\pm 0.013\end{array}$ & & \\
\hline & \multirow{2}{*}{$\mathrm{C} / \mathrm{E}$} & MCNP & 0.976 & 0.997 & & \\
\hline & & $\mathrm{MC}^{2}-3$ & 0.976 & 0.998 & & \\
\hline
\end{tabular}

a) MCNP results are from Reference 55

b) $\mathrm{MC}^{2}-3$ rows denotes results from $\mathrm{MC}^{2}-3 / \mathrm{TWODANT}$ calculation

Table 4. Summary of Eigenvalue Results for Takeda Benchmark 1

\begin{tabular}{c|c|c|c}
\hline & Control Rod In & $\begin{array}{c}\text { Control Rod } \\
\text { Out }\end{array}$ & $\begin{array}{c}\text { Control Rod } \\
\text { Worth }\end{array}$ \\
\hline Reference & $\begin{array}{c}0.96240 \pm \\
0.00071\end{array}$ & $\begin{array}{c}0.97760 \pm \\
0.00069\end{array}$ & $\begin{array}{c}0.01616 \pm \\
0.00099\end{array}$ \\
\hline VARIANT & 0.96240 & 0.97699 & 0.01552 \\
\hline PN2ND & 0.96239 & 0.97649 & 0.01501 \\
\hline SN2ND & 0.96245 & 0.97717 & 0.01565 \\
\hline MOCFE & 0.96171 & 0.97686 & 0.01613 \\
\hline
\end{tabular}

hexahedral meshes, one of which is shown in Fig. 13 PN2ND and SN2ND eigenvalue solutions are summarized in Tables 7 and 8, respectively. The asymptotic convergence toward the reference solution is clearly seen for both PN2ND and SN2ND solutions, but a residual of $\sim 50 \mathrm{pcm}$ is observable in each of the two codes. A similar residual was observed in the VARIANT solution obtained with a $\mathrm{P}_{7}$ angular approximation and a spatial approximation of eighth-, fourth- and third-order polynomials for flux, source, and leakage, respectively, which are usually sufficient for most problems. The residual error is associated with the leakage and source approximation in addition to the angular variable (primary source). In previous work [61], it has 
Table 5. Summary of Eigenvalue Results for Takeda Benchmark 4

\begin{tabular}{c|c|c|c}
\hline & Control Rod In & $\begin{array}{c}\text { Control Rod } \\
\text { Half }\end{array}$ & $\begin{array}{c}\text { Control Rod } \\
\text { Out }\end{array}$ \\
\hline Reference & $\begin{array}{c}0.88001 \pm \\
0.00038\end{array}$ & $\begin{array}{c}0.98340 \pm \\
0.00039\end{array}$ & $\begin{array}{c}1.09515 \pm \\
0.00040\end{array}$ \\
\hline VARIANT & 0.88009 & 0.98415 & 1.09636 \\
\hline PN2ND & 0.87960 & 0.98365 & 1.09599 \\
\hline SN2ND & 0.87877 & 0.98275 & 1.09494 \\
\hline MOCFE & 0.87796 & 0.98164 & 1.09353 \\
\hline & Half Control Rod Worth & Full Control Rod Worth \\
\hline Reference & $0.10376 \pm 0.00056$ & $0.22323 \pm 0.00054$ \\
\hline VARIANT & 0.10400 & & 0.22414 \\
\hline PN2ND & 0.10420 & & \multicolumn{3}{c}{0.22446} \\
\hline SN2ND & 0.10426 & 0.22453 \\
\hline MOCFE & 0.10423 &
\end{tabular}

Table 6. Spatial Refinement Results of MOCFE for Takeda Benchmark 4

\begin{tabular}{c|c|c|c}
\hline $\begin{array}{c}\text { Number of } \\
\text { Elements }\end{array}$ & $\begin{array}{c}\text { Control } \\
\text { Rod In }\end{array}$ & $\begin{array}{c}\text { Control Rod } \\
\text { Half }\end{array}$ & $\begin{array}{c}\text { Control Rod } \\
\text { Out }\end{array}$ \\
\hline 85,538 & 1496 & 1472 & 1474 \\
\hline 194,833 & 919 & 872 & 854 \\
\hline 386,650 & 662 & 596 & 575 \\
\hline 897,248 & 426 & 346 & 329 \\
\hline $2,113,902$ & 310 & 223 & 209 \\
\hline $3,448,832$ & 261 & 176 & 162 \\
\hline
\end{tabular}

${ }^{*}$ Errors in pcm from reference solutions

been shown that all such errors in VARIANT can be removed by full space-angle refinement.

The finite element methodologies in PN2ND and SN2ND have made space-angle refinement much more difficult. The SN2ND results indicate angular convergence using the $\mathrm{S}_{10}$ Carlson even-moment cubature, but clearly residual error is still present. Unlike the VARIANT code, with the finite element methodology, the mesh must be carefully refined near the steep gradients observed in the even-parity flux solution (or the large discontinuities observed in the odd-parity flux solution). Such mesh refinement is tedious and typically leads to many more spatial degrees of freedom than an equivalent VARIANT
Table 7. PN2ND Eigenvalue Solutions for ABTR Benchmark

\begin{tabular}{c|c|c|c}
\hline \multirow{2}{*}{$\begin{array}{c}\text { Angular } \\
\text { Order }\end{array}$} & \multicolumn{3}{|c}{ Spatial Mesh Approximation (vertices) } \\
\cline { 2 - 4 } & 78243 & 113873 & 461219 \\
\hline $\mathrm{P}_{1}$ & -1660 & -1655 & -1594 \\
\hline $\mathrm{P}_{3}$ & -366 & -359 & -249 \\
\hline $\mathrm{P}_{5}$ & -254 & -246 & -109 \\
\hline $\mathrm{P}_{7}$ & -228 & -219 & -70 \\
\hline $\mathrm{P}_{9}$ & -220 & -211 & -55 \\
\hline $\mathrm{P}_{11}$ & -217 & & \\
\hline
\end{tabular}

Table 8. SN2ND Eigenvalue Solutions for ABTR Benchmark

\begin{tabular}{c|c|c|c|c|c}
\hline \multirow{2}{*}{$\begin{array}{c}\text { Angular } \\
\text { Directions }\end{array}$} & \multicolumn{5}{|c}{ Spatial Mesh Approximation (vertices) } \\
\cline { 2 - 6 } & 78243 & 113873 & 461219 & 671219 & 785801 \\
\hline 32 & -241 & -233 & -69 & -64 & -59 \\
\hline 50 & -220 & -210 & -47 & -40 & -37 \\
\hline 72 & -225 & -217 & -51 & & \\
\hline 98 & -216 & -207 & -43 & & \\
\hline 288 & -216 & & & & \\
\hline
\end{tabular}

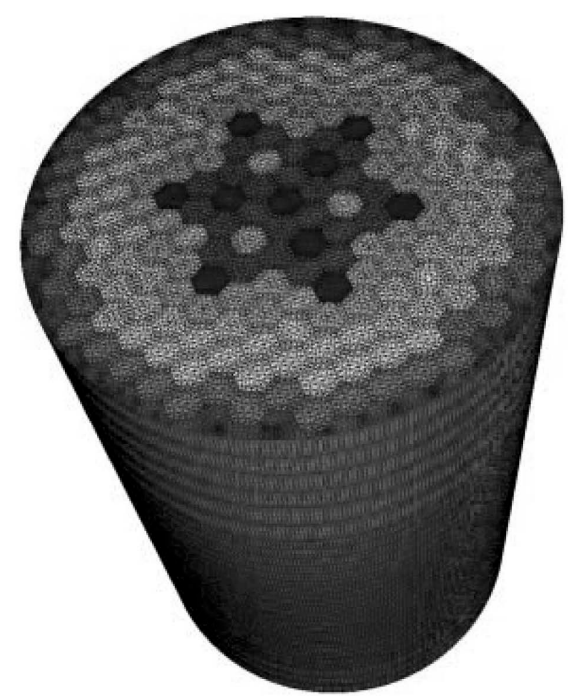

Fig. 13. . Hexahedron Mesh Used for ABTR Benchmark 
Table 9. Multiplication Factors of ZPPR-15A Experiments

\begin{tabular}{c|c|c|c|c}
\hline Loading & Experiment $^{\text {a) }}$ & VIM & MC $^{2}-2 /$ SDX/DIF3D & MC $^{2}-3 / \mathrm{DIF} 3 \mathrm{D}$ \\
\hline 15 & 1.00046 & $0.99985 \pm 0.00020$ & $0.99593(-453)$ & $0.99740(-306)$ \\
\hline 16 & 0.99627 & $0.99571 \pm 0.00021$ & $0.99178(-449)$ & $0.99327(-300)$ \\
\hline 20 & 0.99853 & $0.99742 \pm 0.00019$ & $0.99426(-427)$ & $0.99550(-303)$ \\
\hline
\end{tabular}

a) A maximum uncertainty of $\sim 180$ pcm was estimated for the experimental values.

b) Numbers in parenthesis indicate pcm difference from experimental values.

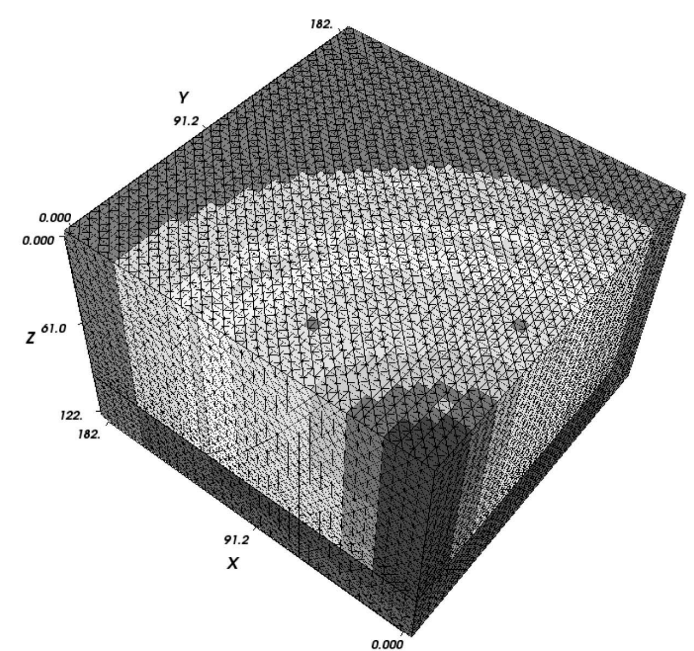

Fig. 14. Geometrical Layout of ZPPR-15A (Octant Core)

calculation. Combined with the advantages of the structured geometry treatment over an unstructured one, the PN2ND and SN2ND solvers require more computational time on assembly homogenized problems, and in many cases can require several thousand processors to produce the same solution in a comparable time. From this study, it is concluded that the finite element-based SN2ND and PN2ND solvers are not the most efficient methodologies for treating assembly homogenized problems, especially when compared with a traditional nodal approach. However, nodal formulations typically become less attractive for smaller mesh sizes and require error-prone homogenization and dehomogenization techniques, which complicate multiphysics coupling.

\subsection{ZPR-6 and ZPPR Critical Experiments}

Combined performance of $\mathrm{MC}^{2}-3$ and UNIC was tested against the ZPR-6 and ZPPR critical experiments. The onedimensional ultrafine group transport capability of $\mathrm{MC}^{2}-3$
Table 10. PN2ND Multiplication Factors for ZPPR-15A Loading 15 (ENDF/B-V.2 Data)

\begin{tabular}{c|c|c}
\hline $\begin{array}{c}\text { Angular Flux } \\
\text { Expansion Order }\end{array}$ & $\begin{array}{c}\text { Scattering } \\
\text { Order }\end{array}$ & $\begin{array}{c}\text { Multiplication } \\
\text { Factor }\end{array}$ \\
\hline $\mathrm{P}_{1}$ & $\mathrm{P}_{1}$ & 0.99258 \\
\hline $\mathrm{P}_{3}$ & $\mathrm{P}_{3}$ & 0.99640 \\
\hline $\mathrm{P}_{5}$ & $\mathrm{P}_{5}$ & 0.99651 \\
\hline \multicolumn{2}{|c|}{ VIM Monte Carlo } & $0.99616 \pm 0.00010$ \\
\hline
\end{tabular}

was first tested by analyzing the three loadings of ZPPR-15A and comparing the results to those of a previous study [50]. Figure 14 shows the geometrical layout for the ZPPR-15A Loading 15. Cell-averaged drawer cross sections were generated based on the ENDF/B-VII.0 data in a 230-group structure using the 1-D transport capability of $\mathrm{MC}^{2}-3$, while in the previous study they were generated based on the ENDF/V.2 and ENDF/VII.0 data using the $\mathrm{MC}^{2}-2$ and SDX codes. To compare with the previous results, the core calculations were performed with the DIF3D nodal transport option [62], whose accuracy is comparable to $\mathrm{S}_{4}$ discrete ordinate solutions with $\mathrm{P}_{1}$ scattering approximation. It is noted that the VARIANT option cannot be used for these calculations because the $\sim 5 \mathrm{~cm}$ node leads to convergence problems. (While these issues can be fixed, it is not the focus of our development.)

Table 9 compares the calculated multiplication factors with the experimental values and VIM Monte Carlo solutions. The VIM Monte Carlo solutions were obtained using the as-built plate-by-plate models. It can be seen that $\mathrm{MC}^{2}-3$ cross sections improve the DIF3D solutions by $\sim 150$ pcm relative to $\mathrm{MC}^{2}-2 / \mathrm{SDX}$ cross sections. However, $\mathrm{MC}^{2}-3 / \mathrm{DIF} 3 \mathrm{D}$ solutions still underestimate the multiplication factor by $\sim 300 \mathrm{pcm} \Delta \mathrm{k}$ compared to VIM. A previous, unpublished study with the PN2ND solver and ENDF/B-V.2 data showed that the multiplication factor 
of the ZPPR-15A Loading 15 almost converged using a $\mathrm{P}_{3}$ angular flux approximation and a $\mathrm{P}_{3}$ scattering order as shown in Table 10. It can be seen that the multiplication factor increases by $\sim 400 \mathrm{pcm}$ when the angular approximation order for flux and scattering is increased to $P_{3}$ from $P_{1}$. Thus, it is expected that the calculated multiplication factor would be close to the experimental value, when the higher order angular approximations are applied.

To validate the accuracy of the SN2ND solver in addition to the cross section generation algorithms of $\mathrm{MC}^{2}-3$, the uranium-fueled ZPR-6/6A and the high Pu-240 core of ZPR-6/7 that includes a high Pu-240 zone at the center of the plutonium-fueled core were analyzed. The ZPR-6/6A assembly was slightly simplified by modeling similar drawers by a representative one, whereas the as-built models were used for ZPR- $6 / 7$ by modeling more than 100 drawer types explicitly. Cell-averaged drawer cross sections were generated based on the ENDF/B-VII.0 data in 9-, 33-, 70-, 116-, and 230-group structures using the 1-D transport capability of $\mathrm{MC}^{2}-3$. Several meshes and angular cubatures were implemented at each energy resolution.

For ZPR-6/6A, it was determined that a mesh with about 470,000 vertices and an $S_{6}$ angular cubature provided sufficient space-angle convergence (within $10 \mathrm{pcm}$ ). The reference continuous-energy VIM Monte Carlo solution of the plate-by-plate heterogeneous problem was 0.99981 \pm 0.00025 . The SN2ND solutions obtained with 9-, 33-, $116-$, and 230-energy groups showed eigenvalue errors of $26,-15,-16$, and $-15 \mathrm{pcm}$, respectively. That is, after an initial adjustment of $41 \mathrm{pcm}$ from 9- to 33-energy groups, no further changes are observed in as many as 230 groups. Since these eigenvalues were generated with full spaceangle convergence (496,496 quadratic vertices and 48 discrete ordinates), these results successfully indicate the accuracy of $\mathrm{MC}^{2}-3$ and SN2ND.

Table 11 summarizes the SN2ND eigenvalue results for the loading 104 of ZPR-6/7. The results were obtained using the PN2ND solver in diffusion theory (with transport cross sections provided by $\mathrm{MC}^{2}-3$ ). The experimental value was $1.00072 \pm 0.00085$, where the uncertainty was estimated by adding the measurement uncertainty of $4.6 \mathrm{pcm}$ to the combined uncertainty of $80.4 \mathrm{pcm}$ for geometry and composition uncertainties. The results in Table 11 show the convergence of SN2ND solutions with respect to energy, mesh, and angular cubature, along with the importance of anisotropic scattering. Hexahedral meshes were used for all of these calculations where the 57,132 vertex mesh is considered a test case since it uses linear hexahedral trial functions. The 223,928 vertex mesh assumes $\sim 8 \mathrm{~cm}$ axial element sizes and one hexahedron per homogenized drawer. The 805,185 vertex mesh also uses $\sim 8 \mathrm{~cm}$ axial element sizes but it defines 4 hexahedrons per homogenized drawer, which yields about 9 pcm compared with the 223,928 vertex mesh. The 1,467,429 vertex mesh also uses 4 hexahedrons per homogenized drawer but targets a $\sim 5 \mathrm{~cm}$ axial mesh size which yields no significant improvement over the 805,185 vertex mesh. In general, the 805,185 vertex mesh is considered sufficiently refined for our needs. The angular cubature has virtually no impact on this homogeneous core, but a $\sim 40 \mathrm{pcm}$ error associated with anisotropic scattering occurs. No attempt was made to use $\mathrm{P}_{5}$ anisotropic scattering, but experience indicates that not much difference exists between $\mathrm{P}_{3}$ and $\mathrm{P}_{5}$ scattering on these types of problems. The convergence with respect to energy is quite curious because the 70- and 230-group solutions appear identical while the 116-group result is not consistent. This same behavior was not observed in the loading 106 results shown below, but it is worth investigating in the future.

Table 11. SN2ND and Diffusion Multiplication Factors for ZPR-6/7 Loading 104

\begin{tabular}{|c|c|c|c|c|c|c|c|}
\hline \multirow{2}{*}{$\begin{array}{c}\text { Mesh } \\
\text { Vertices }\end{array}$} & \multirow{2}{*}{$\begin{array}{c}\text { Angular } \\
\text { Directions }\end{array}$} & \multirow{2}{*}{$\begin{array}{c}\text { Scattering } \\
\text { Order }\end{array}$} & \multicolumn{5}{|c|}{ Number of Energy Groups } \\
\hline & & & 9 & 33 & 70 & 116 & 230 \\
\hline 57,132 & 32 & $\mathrm{P}_{1}$ & 0.99676 & 0.99828 & 0.99905 & 0.99880 & 0.99905 \\
\hline 223,928 & 32 & $P_{1}$ & 0.99845 & 1.00001 & 1.00080 & 1.00055 & 1.00081 \\
\hline 805,185 & 32 & $\mathrm{P}_{1}$ & 0.99854 & 1.00010 & 1.00089 & 1.00064 & 1.00090 \\
\hline 805,185 & 32 & $\mathrm{P}_{3}$ & 0.99888 & 1.00044 & 1.00123 & & \\
\hline 805,185 & 72 & $P_{1}$ & 0.99853 & 1.00009 & 1.00088 & 1.00063 & 1.00089 \\
\hline 805,185 & 72 & $\mathrm{P}_{3}$ & 0.99887 & 1.00044 & 1.00122 & & \\
\hline $1,467,429$ & 32 & $P_{1}$ & 0.99855 & 1.00011 & 1.00090 & 1.00065 & 1.00092 \\
\hline $1,467,429$ & Diffusion & $\mathrm{P}_{0}$ & 0.99911 & 1.00065 & 1.00145 & 1.00105 & 1.00130 \\
\hline
\end{tabular}

${ }^{*}$ Experimental value $=1.00072 \pm 0.00085$ 
The reaction rate distributions measured with activation foils were also calculated by superimposing the local flux shapes from $\mathrm{MC}^{2}-3$ to the global flux distributions from SN2ND. Figure 15 shows a preliminary result for the normalized radial distributions of enriched uranium (EU) fission, depleted uranium (DU) capture and fission, and $\mathrm{Pu}-239$ fission reactions. All data are arbitrarily normalized to unity at the $66.2 \mathrm{~cm}$ radial position. The maximum deviation of the calculated values ( $\mathrm{C}$ in Fig. 15) from the experimental values ( $\mathrm{E}$ in Fig. 15) was $2.7 \%$ for EU fission, $1.7 \%$ for DU capture, $3.5 \%$ for DU fission, and 3.4\% for $\mathrm{Pu}-239$ fission, while the estimated measurement uncertainties were $1.6,2.0,2.8$, and $1.5 \%$, respectively.

Table 12 summarizes the SN2ND eigenvalue results for the loading 106 of ZPR-6/7. The experimental value was $1.00091 \pm 0.00086$, where the uncertainty was determined by adding the measurement uncertainty of 5.8 pcm to the combined uncertainty of 80.4 pcm for geometry and composition uncertainties. The continuous-energy Monte Carlo solution obtained from MCNP5 was $1.00049 \pm$ 0.00007. Again, it can be seen that the SN2ND solution converges with refined energy, mesh, angular cubature and scattering order. Figure 16 shows some selected flux plots from the 70-group calculation of the loading 106 of ZPR-6/7. As shown in Fig. 10, beryllium oxide was loaded in the four drawers surrounding the sodium drawer at the core center to enhance the worth of the simulated $\mathrm{B}_{4} \mathrm{C}$ control rod. This causes significant localized flux changes with respect to those drawers without beryllium. For each group plot in Fig. 16, the right hand picture displays the face of the movable matrix half as displayed in Fig. 10, while the left hand picture shows the flux solution for the active core of the stationary side (everything inside the depleted uranium blanket). For the stationary side, the high $\mathrm{Pu}-240$ zone is separated to display the axial flux solution along with the central BeO modified drawers, allowing the axial flux variation to be observed. As can be seen, the $\mathrm{BeO}$ drawers yield only modest impacts on the high-energy and unresolved resonance regions. In the epithermal range, a substantial peak is observed in the flux solution due to the improved scattering source derived from the $\mathrm{BeO}$ drawer. To validate the calculated flux

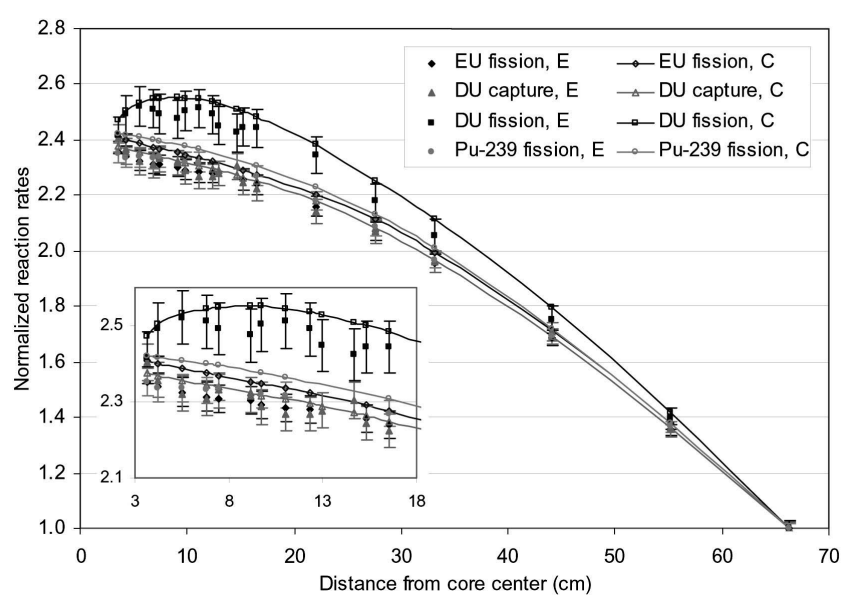

Fig. 15. Radial Distributions of Reaction Rates for Loading 104 of ZPR-6/7

Table 12. SN2ND Multiplication Factors for ZPR-6/7 Loading 106

\begin{tabular}{c|c|c|c|c|c|c|c}
\hline \multirow{2}{*}{$\begin{array}{c}\text { Mesh } \\
\text { Vertices }\end{array}$} & \multirow{2}{*}{$\begin{array}{c}\text { Angular } \\
\text { Directions }\end{array}$} & \multirow{2}{*}{$\begin{array}{c}\text { Scattering } \\
\text { Order }\end{array}$} & \multicolumn{5}{|c}{ Number of Energy Groups } \\
\cline { 4 - 7 } & 32 & $\mathrm{P}_{1}$ & 0.99739 & 0.99920 & 0.99954 & 0.99973 & 230 \\
\hline 103,684 & 32 & $\mathrm{P}_{1}$ & 0.99851 & 1.00037 & 1.00071 & 1.00091 & 1.00123 \\
\hline 299,276 & 32 & $\mathrm{P}_{1}$ & 0.99852 & 1.00037 & 1.00071 & 1.00092 & 1.00123 \\
\hline 408,112 & 32 & $\mathrm{P}_{3}$ & 0.99887 & 1.00072 & 1.00105 & 1.00126 & 1.00157 \\
\hline 408,112 & 72 & $\mathrm{P}_{1}$ & 0.99851 & 1.00036 & 1.00070 & 1.00091 & 1.00122 \\
\hline 408,112 & 72 & $\mathrm{P}_{3}$ & 0.99886 & 1.00070 & 1.00105 & 1.00125 & 1.00060 \\
\hline 408,112 & 32 & $\mathrm{P}_{1}$ & 0.99822 & 1.00006 & 1.00040 & 1.00091 \\
\hline $1,001,437$ & 32 & $\mathrm{P}_{3}$ & 0.99856 & 1.00039 & 1.00074 & 1.00093 & 1.00060 \\
\hline $1,001,437$ & 72 & $\mathrm{P}_{1}$ & 0.99822 & 1.00006 & 1.00040 & 1.00091 \\
\hline $1,001,437$ & 72 & $\mathrm{P}_{3}$ & 0.99856 & 1.00040 & & 1.00094 & \\
\hline $1,001,437$ & 1.00091 & 0.00086 & & & \\
\hline
\end{tabular}

${ }^{*}$ Experimental value $=1.00091 \pm 0.00086 ;$ MCNP5 result $=1.00049 \pm 0.00007$ 


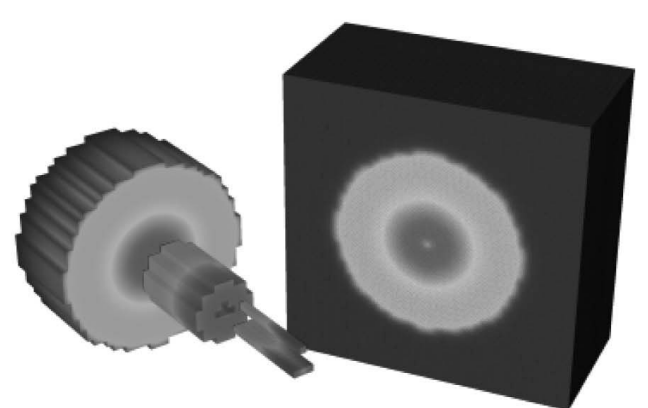

Group 1 of $70(10 \mathrm{MeV}-14.19 \mathrm{MeV})$

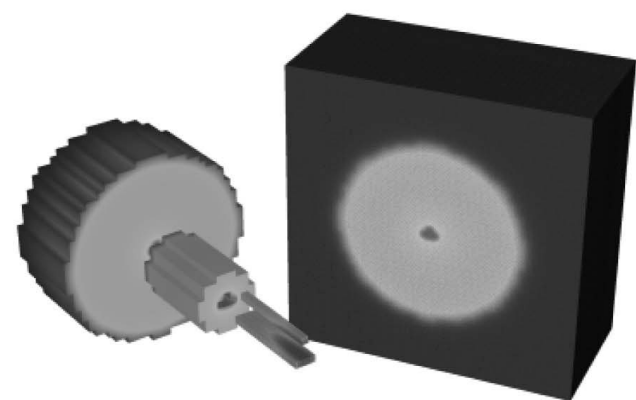

Group 30 of $70(7102 \mathrm{eV}-9119 \mathrm{eV})$

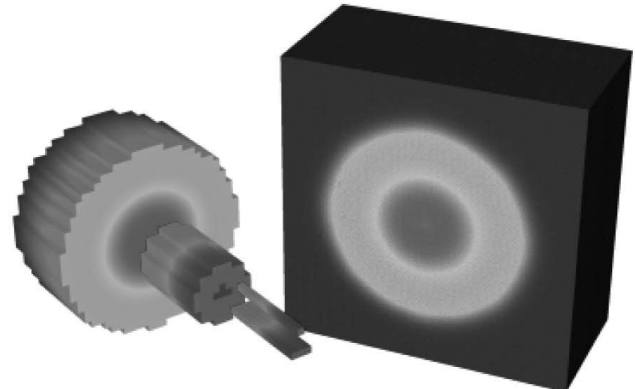

Group 15 of $70(302 \mathrm{keV}-387 \mathrm{keV})$

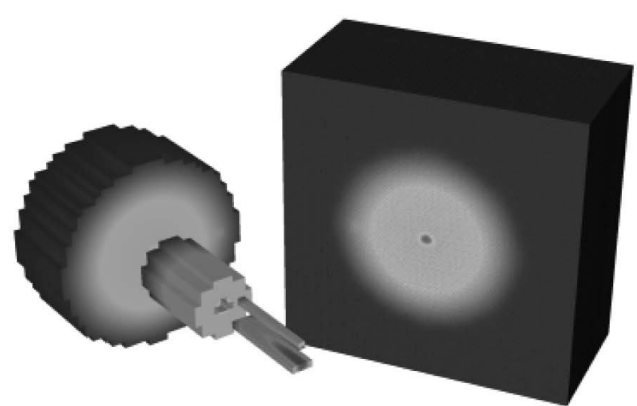

Group 44 of $70(214 \mathrm{eV}-275 \mathrm{eV})$

Fig. 16. Selected Flux Plots for Loading 106 of ZPR-6/7

distribution, the reaction rate distributions measured with activation foils are being analyzed.

\subsection{Parallel Computation Performance}

All three solvers of UNIC can run on small- and largescale parallel machines. The PN2ND, MOCFE, and SN2ND solvers have been executed on thousands, tens of thousands, and hundreds of thousands of processors, respectively, but the parallel performances of PN2ND and MOCFE have not been studied systematically. Thus, this subsection focuses on the parallel performance of the SN2ND solver, which recently merited finalist consideration for the 2009 Gordon Bell Supercomputing Prize [63]. This parallel performance study was performed using a plateby-plate model of ZPR-6/6A. Since the goal was to investigate parallel performance, drawer-averaged cross sections were used rather than regenerating plate-wise cross sections for all drawer types included in the assembly.

With regard to parallel calculations, two measures of performance are typically considered: strong and weak scalabilities. Strong scalability shows how well time-tosolution can be reduced by increasing the number of processors. Weak scalability shows how efficiently the methodology can solve ever larger problems with proportionally larger processor counts. The weak and strong scalabilities of SN2ND were studied on three high-performance computers. Two of the machines are the IBM BlueGene/P architectures hosted by ANL (40 racks with a total of 163,840 cores) [37] and by Jülich Supercomputing Center (JSC) in Germany ( 72 racks with a total of 294,912 cores) [64]. The third machine is the Cray XT5 hosted by Oak Ridge National Laboratory (225,000 cores) [65].

The Oak Ridge machine has the fastest individual processors and highest memory per core of the three machines, but the network speed on the IBM machine is notably more efficient for our calculations. Because of the low memory capabilities of the IBM machines, the scalability studies were generally limited to 9 or 33 energy groups, although larger group calculations were performed using as many as 116 groups but with coarser space-angle representations. Because UNIC can handle parallelization by space, angle, and energy, strong and weak scalabilities can be considered for each variable both separately and together, but this analysis was restricted to parallelization by space and angle separately.

Table 13 shows the strong scalability results in space on Blue Gene/P. A 15 million vertex mesh was used with 1.8 million hexahedral elements, a 9-group cross-section set, and an $\mathrm{S}_{6}$ angular cubature. Because of the memory 
limitations on BlueGene/P (512 MB per core), it was difficult to get a large number of vertices per core, and thus this study was performed by spreading the mesh over 4,096 cores yielding 7,324 vertices per core. The mesh was further partitioned over 8 times as many processors leading to $\sim 1,831$ vertices per process. Below 800 vertices per process, extremely diminished performance was typically seen due to the increased communication and iteration requirements. This type of behavior is not unusual for the SSOR preconditioner used for the conjugate gradient solver in PETSc (where the iteration count increases as the space gets partitioned over more subdomains), and thus it is not necessarily indicative of the parallel machine or the SN2ND methodology. Note that the set of angular directions is spread into two sets of 4,096 cores. Each such set has the mesh replicated, thus giving a hierarchical division of work.

Because the synthetic diffusion equation has not been partitioned over the full space-angle set of processors yet, the strong scalability in angle $(<75 \%)$ is not as good as that in space. Future development is focused on distributing the diffusion system and implementing spatial multigrid methods such that the number of vertices per core can be increased and the memory problems currently being experienced can be reduced. Spatial multigrid, when effective, has been shown to maintain the high scalability shown above when applied to similar equations to those solved by SN2ND.

Because space-angle convergence studies often necessitate increasing space-angle resolutions until a desired accuracy is achieved, weak scaling studies and performance are more important than strong scalability. In fact, several of the ZPR-6 calculations employed the maximum number of processors and memory available on the parallel machines, despite the fact that none of these studies had indicated full space-angle convergence (even without considering parallelization by energy). Table 14 gives the weak scaling performance for calculation with 9 energy groups on Blue Gene/P. As can be seen, the performance is excellent up to 131,072 cores, but beyond this a rapid drop-off is observable. An inspection of the timing history indicates that this is mostly attributable to a breakdown in performance of the "global reduce" operation associated with the within-group scattering source update. This operation on user-defined communicators is not expected to be implemented optimally in vendor-supplied MPI libraries. Currently, various approaches to mapping MPI processes to cores are being explored to obtain better performance for high core count cases.

Table 15 gives the weak angle scalability of SN2ND on XT5 for a calculation with 33 energy groups. Similar to the BlueGene/P results, it shows an observable drop in performance, but this time it occurs at 100,000 cores, which is associated with the different network characteristics of the two machines. However, it is important to note that the faster processor speed and larger memory per core of the
Table 13. Strong Spatial Scalability of SN2ND on BlueGene/P at ANL

\begin{tabular}{c|c|c|c}
\hline $\begin{array}{c}\text { Total } \\
\text { Cores }\end{array}$ & $\begin{array}{c}\text { Vertices } \\
\text { Per Process }\end{array}$ & $\begin{array}{c}\text { Total } \\
\text { Time }(\mathrm{sec})\end{array}$ & $\begin{array}{c}\text { Strong } \\
\text { Scaling }\end{array}$ \\
\hline 8,192 & 7,324 & 2,402 & $100 \%$ \\
\hline 16,384 & 3,662 & 1,312 & $92 \%$ \\
\hline 24,576 & 2,441 & 873 & $92 \%$ \\
\hline 32,768 & 1,831 & 637 & $94 \%$ \\
\hline
\end{tabular}

Table 14. Weak Angle Scalability of SN2ND on BlueGene/P (Combined ANL and JSC)

\begin{tabular}{c|c|c|c}
\hline $\begin{array}{c}\text { Total } \\
\text { Cores }\end{array}$ & $\begin{array}{c}\text { Angular } \\
\text { Directions }\end{array}$ & $\begin{array}{c}\text { Total } \\
\text { Time }(\mathrm{sec})\end{array}$ & $\begin{array}{c}\text { Weak } \\
\text { Scaling }\end{array}$ \\
\hline 32,768 & 32 & 579 & $100 \%$ \\
\hline 73,728 & 72 & 572 & $101 \%$ \\
\hline 131,072 & 128 & 581 & $100 \%$ \\
\hline 163,840 & 160 & 691 & $84 \%$ \\
\hline 294,912 & 288 & 763 & $76 \%$ \\
\hline
\end{tabular}

Table 15. Weak Angle Scalability of SN2ND on XT5 (ORNL)

\begin{tabular}{c|c|c|c}
\hline $\begin{array}{c}\text { Total } \\
\text { Cores }\end{array}$ & $\begin{array}{c}\text { Angular } \\
\text { Directions }\end{array}$ & $\begin{array}{c}\text { Total } \\
\text { Time }(\mathrm{sec})\end{array}$ & $\begin{array}{c}\text { Weak } \\
\text { Scaling }\end{array}$ \\
\hline 16,512 & 32 & 1891 & $100 \%$ \\
\hline 37,152 & 72 & 1901 & $99 \%$ \\
\hline 66,048 & 128 & 1829 & $103 \%$ \\
\hline 103,200 & 200 & 2050 & $92 \%$ \\
\hline 148,608 & 288 & 2298 & $82 \%$ \\
\hline 222,912 & 432 & 2517 & $75 \%$ \\
\hline
\end{tabular}

XT5 machines allows the 33-group problem to be solved in a comparable amount of time to the 9-group calculation on Blue Gene/P. Using XT5, it was possible to obtain ZPR$6 / 6 \mathrm{~A}$ results containing over 0.9 trillion degrees of freedom. 
For spatial weak scaling, the previous calculations with assembly-homogenized problems indicated weak scalability between 70 and $80 \%$, but these numbers do not extend past a few thousand processors. For the plate-by-plate heterogeneous problems used in this study, the required degree of mesh refinement is somewhat fixed because of the physics in the problem (optical thickness of regions). Unlike the assembly-homogenized problems, mesh refinement in heterogeneous problems can easily change the condition number of the coefficient matrix and thus pollute the timing data. As an example, finite elements can be defined with very bad aspect ratios that yield a poor coefficient matrix condition number. More vertices introduced with mesh refinement would typically translate to a decreasing weak scalability, but in our case with axial mesh refinement, it generally improves the condition number of the coefficient system so that it appears to obtain greater than $100 \%$ weak scaling. However, if the mesh is artificially refined past the point of asymptotic convergence, the condition number becomes worse and it appears to have very poor weak scaling. Neither of these scenarios tells much about the actual weak scaling performance of the coding. In the future, it will be necessary to devise contrived heterogeneous benchmark problems that retain the same physics properties but allow the domain size to be increased in some regular fashion.

\section{CONCLUSION}

As part of U.S. DOE's NEAMS program, ANL is developing a suite of modern fast reactor simulation tools. The general goal is to reduce the uncertainties and biases in various areas of reactor design activities by providing enhanced prediction capabilities. Under this fast reactor simulation program, a high-fidelity deterministic neutron transport code UNIC and a multigroup cross section generation code $\mathrm{MC}^{2}-3$ are being developed. The end goal of this development is to produce an integrated neutronics code that enables the high-fidelity description of a nuclear reactor and simplifies the multistep design process by direct and accurate coupling with thermal hydraulics and structural mechanics calculations.

The application scope targeted for UNIC ranges from the homogenized assembly approaches prevalent in current reactor analysis methodologies to explicit geometry, timedependent transport calculations that are directly coupled to thermal-hydraulics and structural mechanics calculations in reactor accident simulations. Three transport equation solvers have been developed based upon the unstructured finite-element mesh representation: PN2ND, SN2ND, and MOCFE. PN2ND and SN2ND solve the second-order even-parity transport equation with spherical harmonics and discrete ordinates angular approximations, respectively. MOCFE is a method of characteristics solver for the firstorder transport equation, of which targeted application is for explicit geometry, fine-group problems. The nodal transport code VARIANT is also being rebuilt in UNIC as NODAL which is focused on solving modern assembly homogenized problems. To generate multigroup cross sections in fast reactor applications, $\mathrm{MC}^{2}-3$ has been developed by improving the resonance self-shielding and spectrum calculation methods of $\mathrm{MC}^{2}-2$, and by integrating the one-dimensional cell calculation capabilities of SDX.

The solution methods and modeling capabilities of the three transport solvers of UNIC were briefly discussed. The finite element discretization of spatial domain and the spherical harmonics and discrete ordinates angular approximations implemented in UNIC solvers were discussed along with the iterative solution methods for the resulting system of discretized equations. The spaceangle-energy domain decomposition approach for massively parallel computations was also discussed. It is noted that SN2ND can already be used on both the world's largest and the world's fastest machines (Top 500 in November 2009) using space-angle partitioning alone. The slowingdown calculation methods of $\mathrm{MC}^{2}-3$ were also discussed, focusing on enhanced methods for resonance self-shielding and spectrum calculations.

The performances of $\mathrm{MC}^{2}-3$ and UNIC were tested using several well known benchmark problems and fast critical experiments. The $\mathrm{MC}^{2}-3$ results for homogeneous medium and unit cell calculations and the transport solutions obtained with $\mathrm{MC}^{2}-3$ cross sections for simplified benchmark problems derived from fast critical experiments were compared to MCNP and VIM Monte Carlo solutions. The results indicated that $\mathrm{MC}^{2}-3$ produces accurate multigroup cross sections for homogeneous media and heterogeneous unit cells. The UNIC calculations for threedimensional homogenized benchmark problems with given sets of group cross sections showed that for all three solvers, asymptotic convergence toward the reference multigroup Monte Carlo solutions was observable. This paper also discussed the fact that for assembly-homogenized problems, the performance of nodal spatial discretization is quite superior in performance and accuracy to a low order finite element method. Combined tests of $\mathrm{MC}^{2}-3$ and UNIC for the ZPR-6 and ZPPR critical experiments also showed that $\mathrm{MC}^{2}-3 / \mathrm{SN} 2 \mathrm{ND}$ calculations are sufficiently accurate for analyzing these experiments. Some timing results for the SN2ND solver on the ZPR-6 critical assembly benchmarks were also discussed. While they can only give a snapshot of the solver performance in this phase of its development, these numbers indicate successful parallel performance, achieving $76 \%$ weak angle scaling on 294,912 cores and $94 \%$ strong spatial scaling on 32,768 cores.

Most of the UNIC calculations were performed on high performance computers unavailable to most reactor designers, and substantial research and development is still required to make UNIC applicable to practical design calculations. The most important research that remains is to develop a better preconditioner on the space-angle system 
along with one on the entire space-angle-energy system, in particular for MOCFE. Other improvement needs for each solver of UNIC were discussed in Sections 2 and 3 while discussing the solution methods and initial test results. Our current efforts are focused on developing a multigrid preconditioner in SN2ND and a HFG transport calculation capability with anisotropic scattering sources in $\mathrm{MC}^{2}-3$. We also are integrating the MOCFE solver into the $\mathrm{MC}^{2}-3$ code to improve the accuracy of the cross section generation, and we are building a nodal transport solver in UNIC for application to assembly homogenized problems.

Our future efforts will include carrying out timedependent multi-physics calculations for full reactor core-sized problems on current and future large-scale architectures, simulating various accident scenarios such as control rod ejections and loss of flow without scram. The higher fidelity solution capability will not only allow us to better understand the behavior of existing reactor systems, but also predict the behavior of many advanced design concepts.

\section{ACKNOWLEDGMENTS}

This work was supported by the U.S. Department of Energy, Office of Nuclear Energy, under contract DEAC02-06CH11357.

\section{REFERENCES}

[1] W. S. Yang and T. A. Taiwo, "Status of Reactor Analysis Methods and Codes in the U.S.A," Proc. of PHYSOR 2004: The Physics of Fuel Cycles and Advanced Nuclear Systems: Global Developments, Chicago, Illinois, April 25-29, 2004.

[2] T. Takeda, "Neutronics Codes Currently Used in Japan for Fast and Thermal Reactor Applications," Proc. of PHYSOR 2004: The Physics of Fuel Cycles and Advanced Nuclear Systems: Global Developments, Chicago, Illinois, April 25-29, 2004.

[ 3 ] Kord Smith, "Needs Related to the Nuclear Power Industry," Proc. of PHYSOR 2004: The Physics of Fuel Cycles and Advanced Nuclear Systems: Global Developments, Chicago, Illinois, April 25-29, 2004.

[4] H. H. Hummel and D. Okrent, Reactivity Coefficients in Large Fast Reactors, American Nuclear Society (1970).

[5] D. C. Wade and E. K. Fujita, "Trends versus Reactor Size of Passive Reactivity Shutdown and Control Performance," Nucl. Sci. Eng., 103, 182 (1989).

[6] P. Fischer, D. Kaushik, D. Nowak, A. Siegel, W. S. Yang, and G. W. Pieper, "Advanced Simulation for Fast Reactor Analysis," SciDAC Review, Fall 2008 (2008).

[ 7 ] G. Palmiotti, M. A. Smith, C. Rabiti, D. Kaushik, A. Siegel, B. Smith, and E. E. Lewis, "UNİC: Ultimate Neutronic Investigation Code," Proc. of Joint Int. Topical Meeting on Mathematics \& Computation and Supercomputing in Nuclear Applications (M\&C+SNA 2007), Monterey, California, April 15-19, 2007.

[ 8 ] M. A. Smith, D. Kaushik, A. Wollaber, W. S. Yang, B. Smith, C. Rabiti, G. Palmiotti, "Recent Research Progress on UNIC at Argonne National Laboratory," Proc. of Int. Conf. on Mathematics, Computational Methods \& Reactor Physics,
Saratoga Springs, New York, May 3-7, 2009.

[9] M. A. Smith, D. Kaushik, A. Wollaber, W. S. Yang, and B. Smith, "New Neutronics Analysis Tool Development at Argonne National Laboratory," Proc. of Int. Conf. on Fast Reactors and Related Fuel Cycles (FRO9), Kyoto, Japan, December 7-11, 2009.

[10] T. D. Blacker et al., "CUBIT Mesh Generation Environment, Volume 1: Users Manual,” SAND94-1100, Sandia National Laboratory (1994).

[11] B. J. Whitlock, "VisIt User's Manual," UCRL-SM-220449, Lawrence Livermore National Laboratory (2005).

[12] C. B. Carrico, E. E. Lewis and G. Palmiotti, "Three Dimensional Variational Nodal Transport Methods for Cartesian, Triangular and Hexagonal Criticality Calculations," Nucl. Sci. Eng. 111, 168 (1992).

[13] G. Palmiotti, E. E. Lewis and C. B. Carrico, "VARIANT: VARIational Anisotropic Nodal Transport for Multidimensional Cartesian and Hexagonal Geometry Calculation," Argonne National Laboratory, ANL-95/40 (1995).

[14] C. H. Lee and W. S. Yang, "Development of Multi-group Cross Section Generation Code $\mathrm{MC}^{2}-3$ for Fast Reactor Analysis," Proc. of Int. Conf. on Fast Reactors and Related Fuel Cycles (FR09), Kyoto, Japan, December 7-11, 2009.

[15] H. Henryson II, B. J. Toppel, and C. G. Stenberg, "MC'-2: A Code to Calculate Fast Neutron Spectra and Multi-group Cross Sections," ANL-8144, Argonne National Laboratory (1976).

[16] B. J. Toppel, H. Henryson II, and C. G. Stenberg, "ETOE2/MC 2 -2/SDX Multi-group Cross-Section Processing," Conf-780334-5, Proc. of RSIC Seminar-Workshop on Multi-group Cross Sections, Oak Ridge, TN, March 1978.

[17] E. E. Lewis and W. F. Miller Jr., Computational Methods of Neutron Transport. Wiley, New York (1984).

[18] B. G. Carlson, "Tables of Equal Weight Quadrature EQn Over the Unit Sphere," LA-4734, Los Alamos Scientific Laboratory (1971).

[19] B. G. Carlson, "Transport Theory: Discrete Ordinates Quadrature Over the Unit Sphere," LA-4554, Los Alamos Scientific Laboratory (1971).

[20] C. P. Thurgood, A. Pollard, and H. A. Becker, "The TN Quadrature Set for the Discrete Ordinates Method," Transactions of the ASME, 117, 1068 (1995).

[21] V. I. Lebedev, and D. N. Laikov, "A Quadrature Formula for the Sphere of the 131st Algebraic Order of Accuracy," Doklady Mathematics, 59, 477 (1999).

[22] F. K. Chan and E. M. O'Neill, "Feasibility Study of a Quadrilateralized Spherical Cube Earth Data Base," EPRF Technical Report 2-75, Computer Sciences Corporation (1975).

[23] M. Tegmark, “An Icosahedron-Based Method for Pixelizing the Celestial Sphere," The Astronomical Journal, 470, L81 (1996).

[24] J. N. Reddy, An Introduction to the Finite Element Method, Second Edition, McGraw-Hill, Boston (1993).

[25] O. C. Zienkiewicz and R. L. Taylor, The Finite Element Method, 4th ed., McGraw-Hill, New York (1989).

[26] Y. Sadd, Iterative Methods for Sparse Linear Systems, Second Edition, Society of Industrial and Applied Mathematics (2003).

[27] R. E. Alcouffe, F. W. Brinkley, D. R. Marr, and R. D. O’Dell, 
“User's Guide for TWODANT: A Code Package for TwoDimensional, Diffusion-Accelerated, Neutral-Particle Transport," LA-10049-M, Los Alamos National Laboratory, 1990.

[28] S. Balay, K. R. Buschelman, W. D. Gropp, D. K. Kaushik, M. G. Knepley, L. C. McInnes, and B. F. Smith, "PETSc home page," http://www.mcs.anl.gov/petsc.

[29] E. E. Lewis, A. Wollaber, A. Marin-Lafleche, M. A. Smith, and W. S. Yang, "Comparison of Krylov and p-Multigrid Solutions of Orthogonal Response Matrix Equations," Trans. Am. Nucl. Soc., 101, 538 (2010).

[30] E. E. Lewis, A. Wollaber, A. Marin-Lafleche, M. A. Smith, and W. S. Yang, "Response Matrix Acceleration Methods Based on Orthogonalization and Domain Decomposition," Trans. Am. Nucl. Soc., 101, 540 (2010).

[31] METIS - Family of Multilevel Partitioning Algorithms, Karypis Lab, http://glaros.dtc.umn.edu/gkhome/views/metis/.

[32] J. R. Askew, "A Characteristics Formulation of the Neutron Transport Equation in Complicated Geometries," AAEWM 1108, United Kingdom Atomic Energy Establishment (1972).

[33] B. G. Carlson, "A Method of Characteristic and Other Improvements in Solution Methods for the Transport Equation," Nucl. Sci. Eng., 61, 408 (1976).

[34] C. Rabiti, M. A. Smith, W. S. Yang, D. Kaushik, and G. Palmiotti, "Parallel Method of Characteristics in Unstructured Finite Element Meshes for the UNIC Code," Proc. of PHYSOR 2008, Interlaken, Switzerland, September 14-19, 2008.

[35] C. Rabiti, G. Palmiotti, W. S. Yang M. A. Smith, and D. Kaushik, "Quasi Linear Representation of the Isotropic Scattering Source for the Method of Characteristics," Proc. of Int. Conf. on Mathematics, Computational Methods \& Reactor Physics, Saratoga Springs, New York, May 3-7, 2009.

[36] T. Möller and B. Trumbore, "Fast, Minimum Storage RayTriangle Intersection," Journal of Graphics tools, 2, 21 (1997).

[37] Argonne Leadership Computing Facility, Argonne National Laboratory, http://www.alcf.anl.gov.

[38] I. I. Bondarenko, et al, Group Constants for Nuclear Reactor Calculations, Consultants Bureau Enterprises, Inc., New York (1964).

[39] M. Segev, "A Theory of Resonance-Group Self-Shielding," Nucl. Sci. and Eng., 56, 72 (1975).

[40] R. E. MacFarlane and D. W. Muir, "The NJOY Nuclear Data Processing System Version 91," LA-12740-M, Los Alamos National Laboratory (1994).

[41] L. B. Levitt, "The Probability Table Method for Treating Unresolved Resonances in Monte Carlo Criticality Calculations," Trans. Am. Nucl. Soc. 14, 648 (1971).

[42] M. N. Nikolaev, et al., "Method of Subgroups for Accounting of Resonance Structure of Cross-sections in Neutron Calculations," Atomn. Energ. 29, 11 (1970).

[43] D. E. Cullen, "Application of the Probability Table Method to Multi-group Calculations of Neutron Transport," Nucl. Sci. Eng. 55, 387 (1974).

[44] P. Ribon and J. M. Maillard, "Les Tables De Probabilite Applications Au Traitement Des Sections Efficaces Pour La Neutronique," Report CEA-N, NEACRP-L-294 (1986).
[45] M. J. Grimstone, J. D. Tullet, and G. Rimpault., “Accurate Treatment of Fast Reactor Fuel Assembly Heterogeneity with the ECCO Cell Code," International Conference on the Physics of Reactors: Operation Design and Computation, PHYSOR 90, April 23-27 (1990).

[46] R. N. Hwang, "A Rigorous Pole Representation of Multilevel Cross Sections and Its Practical Applications," Nucl. Sci. Eng., 96, 192 (1987).

[47] R. N. Hwang, "Efficient Methods for the Treatment of Resonance Integrals,” Nucl. Sci. Eng., 52, 157 (1973).

[48] W. M. Stacey Jr., "Continuous Slowing Down Theory Applied to Fast-Reactor Assemblies," Nucl. Sci. Eng., 41, 381 (1970).

[49] C. H. Lee, W. S. Yang, and R. N. Hill, "Initial Verification and Validation of ENDF/B-VII.0 Libraries of $\mathrm{MC}^{2}-2$ against Fast Critical Systems," Trans. Am. Nucl. Soc., 97, 842 (2007).

[50] S. J. Kim, W. S. Yang, and C. H. Lee, "Analysis of ZPPR15 Critical Experiments with ENDF/B-V.2 and ENDF/BVII.0 Data," Proc. of PHYSOR 2008, Interlaken, Switzerland, September 14-19, 2008.

[51] International Handbook of Evaluated Criticality Safety Benchmark Experiments, NEA/NSC/DOC(95)03, Organization for Economic Co-operation and Development Nuclear Energy Agency (OECD-NEA), September 2009.

[52] International Handbook of Evaluated Reactor Physics Benchmark Experiments, NEA/NSC/DOC (2006)1, Organization for Economic Co-operation and Development Nuclear Energy Agency (OECD-NEA), March 2009.

[53] H. F. McFarlane, et al., "Benchmark Physics Tests in the Metallic-Fueled Assembly ZPPR-15," Nucl. Sci. Eng. 101, 137 (1989).

[54] W. S. Yang and S. J. Kim, Private Communications, Argonne National Laboratory and Korea Atomic Energy Research Institute, September 30, 2009.

[55] M. B. Chadwick et al., "ENDF/B-VII.0: Next Generation Evaluated Nuclear Data Library for Nuclear Science and Technology," Nucl. Data Sheets, 107, 2931 (2006).

[56] F. B. Brown, et al. "MCNP5-1.51 Release Notes," LA-UR09-00384, Los Alamos National Laboratory (2009).

[57] R. N. Blomquist, "VIM Continuous Energy Monte Carlo Transport Code," Proc. Intl. Conf. on Mathematics, Computations, Reactor Physics and Environmental Analysis, Portland, OR, April 30-May 4, 1995.

[58] T. Takeda and H. Ikeda, "3-D Neutron Transport Benchmarks," NEACRP-1-300, Organization of Economic Cooperation and Development - Nuclear Energy Agency (March 1991).

[59] Y. I. Chang, et al., "Advanced Burner Test Reactor Preconceptual Design Report,” ANL-ABR-1 (ANL-AFCI173), Argonne National Laboratory, 2006.

[60] W. S. Yang, T. K. Kim, R. N. Hill, "Core Design Studies for Advanced Burner Test Reactor," Proc. of ICAPP 2007, Nice Acropolis, France, May 13-18, 2007.

[61] M. A. Smith, N. Tsoulfanidis, E. E. Lewis, G. Palmiotti and T. A. Taiwo, "Higher Order Angular Capabilities of the VARIANT Code," Trans. Am. Nucl. Soc., 86, 321 (2002).

[62] R. D. Lawrence, "Progress in Nodal Methods for the Solution of the Neutron Diffusion and Transport Equations," Prog. Nucl. Energy, 17, 271 (1986).

[63] D. Kaushik, M. A. Smith, A. Wollaber, B. Smith, A. Siegel, W. S. Yang, "Enabling High-Fidelity Neutron Transport 
Simulations on Petascale Architectures," Proc. of Int. Conf. for High Performance Computing, Networking, Storage and Analysis, Portland, Or, November 14-20, 2009 (Gordon Bell Award Finalist Paper).
[64] Jülich Supercomputing Center, Jülich Forschungszentrum, http://www.fz-juelich.de/jsc/jugene.

[65] National Center for Computational Sciences, Oak Ridge National Laboratory, http://www.nccs.gov. 